\title{
Effective interaction techniques for the Gamow shell model
}

\author{
Gaute Hagen \\ Department of Physics and Technology, University of Oslo, N-0316 Oslo, Norway; and Centre of Mathematics for Applications, \\ University of Bergen, $N-5007$ Bergen, Norway
}

M. Hjorth-Jensen

Department of Physics and Centre of Mathematics for Applications, University of Oslo, N-0316 Oslo, Norway; PH Division, CERN, CH-1211

Geneva 23, Switzerland; and Department of Physics and Astronomy, Michigan State University, East Lansing, Michigan 48824, USA

Jan S. Vaagen

Department of Physics and Technology, University of Bergen, N-5007 Bergen, Norway

(Received 28 October 2004; published 26 April 2005)

\begin{abstract}
We apply a contour deformation technique in momentum space to the newly developed Gamow shell model, using the drip-line nuclei ${ }^{5} \mathrm{He},{ }^{6} \mathrm{He}$, and ${ }^{7} \mathrm{He}$ as test cases. A major problem in Gamow shell-model studies of nuclear many-body systems is the increasing number of many-body configurations due to the large number of resonant and complex-continuum single-particle orbits necessary to reproduce multiparticle bound and resonant states. We address this problem using two different effective operator approaches generalized to the complex momentum plane. These are the Lee-Suzuki similarity transformation method for complex interactions and the multireference perturbation theory method. We show that a combination of these two approaches results in a drastic truncation of the number of relevant configurations compared with direct diagonalization. This offers interesting perspectives for studies of weakly bound systems.
\end{abstract}

DOI: 10.1103/PhysRevC.71.044314

PACS number(s): 21.60.Cs, 21.10.-k, 24.10.Cn, 24.30.Gd

\section{INTRODUCTION}

Present and proposed nuclear structure research facilities for radioactive beams will open new territory into regions of heavier nuclei. Such systems pose significant challenges to existing nuclear structure models since many of these nuclei will be unstable or short lived. How to deal with weakly bound systems and coupling to resonant states is an open and interesting problem in nuclear spectroscopy. Weakly bound systems cannot be properly described within a standard shell-model approach since even bound states exhibit a strong coupling to the single-particle continuum.

It is therefore important to investigate theoretical methods that will allow the description of systems involved in such element production. Ideally, we would like to start from an $a b$ initio approach with the free nucleon-nucleon interaction and eventually include three-body interactions as the basic building blocks for derivation of an effective shell-model interaction. The newly developed Gamow shell model offers such a possibility; see, for example, Refs. [1-9]. Also, the recently developed shell model embedded in the continuum [10-13] conveys similar interesting perspectives. Here we focus on the Gamow shell model, which promises to be a powerful tool in describing and understanding the formation of multiparticle resonances within a shell-model formulation. Representing the shell-model equations using a Berggren basis [14-19] enables the interpretation of multiparticle resonances in terms of single-particle resonances, as opposed to the traditional harmonic oscillator representation, where resonances never appear explicitly.

Although the Gamow shell-model approach is a powerful tool in this respect, major computational and theoretical challenges need to be overcome if we aim to have a realistic description of weakly bound and unbound nuclei.

One of the first problems encountered in Gamow shellmodel calculations, and addressed in Refs. [4,5], was the problem of identifying the multiparticle resonances on the many-particle energy surface. Studies [4,5] related this identification problem to the problem of choosing a contour in the complex $k$ plane, which in the case of two valence particles selects the interesting physical states from the dense distribution of continuum states. Investigators $[4,5]$ pointed out that when using the classical triangular Berggren contour (see, e.g., Ref. [14]), the unperturbed two-particle energy spectrum would embed the pole configurations in a dense distribution of pole-continuum and continuum-continuum configurations, and an identification based on inspection of the zeroth-order energy spectrum was difficult. As a solution, they employed a "square-well" contour, which in the two-particle case separates the physical states from states belonging to the complex continuum, making such an identification possible. In this way, it is possible to study how the two-particle resonance energy trajectories develop as the nucleon-nucleon interaction is gradually turned on, and in this respect this method is probably the most intuitive. Nevertheless, in the case where more than two particles are present in the shell-model space, the resonant states mix with the complex-continuum states, and an identification becomes difficult.

In Refs. [2,6,7], the problem of identifying multiparticle resonances was approached from a different angle. The proposed algorithm bears close resemblance to the Davidson-Liu $[20,21]$ method. First, a diagonalization within the pole space, where all particles are in resonant single-particle orbitals, is performed. Second, a diagonalization within the complete 
configuration space is performed. Under the assumption of weak coupling of the pole configurations with configurations where at least one particle moves in a continuum orbital, the physical states may be picked out unambiguously from the states obtained after a full diagonalization, using the criterion of largest overlap with the pole space. Also, Refs. [2,6,7] noted that the physical states should be invariant with respect to small deformations of the integration contour, an invariance which may help in identifying the relevant physical states. This method has its strength in that the identification may in principle be carried out for any number of valence particles, under the assumption that the coupling of pole configurations with continuum configurations is weak. On the other hand, this may not always be the case; as pointed out in Ref. [9] for the case of ${ }^{11} \mathrm{Li}$, the two-particle resonances may have a continuum component that is larger than the pole component, depending on the nucleon-nucleon interaction.

In this work, we consider as a test case the light drip-line nuclei ${ }^{5,6,7} \mathrm{He}$ and the formation of resonances in these nuclei starting from a single-particle picture. These nuclei have also been studied with a number of other methods; see, for example, Refs. [22,23] and references therein. Our aim is to establish a methodological test case, where some of the basic physics is already known, and where we may try out both two- and three-body forces. This paper is restricted to two-body forces, and we do not go beyond three valence neutrons. We construct a single-particle basis using the contour deformation method in momentum space, discussed in detail in Ref. [19]; see also Ref. [24] for further references on complex scaling. In our case, the identification problem is solved based on inspection of the unperturbed many-particle energy spectrum, as done in Refs. [4,5]. We show that by choosing a rotated-plus-translated contour in the complex plane, a large portion of the many-particle energy surface is free from complex-continuum states, and the basic pole configurations will never be embedded in the dense continuum. Thus, it is straightforward to study the resonance trajectories as the nucleon-nucleon interaction is turned on. The geometry of the contour is of such a simple form (rotation plus translation) that on the many-particle energy surface the locations of complex energy thresholds and multiparticle resonances are easily identifiable. Our procedure tested for ${ }^{7} \mathrm{He}$ makes a clear distinction of three-particle resonances from the dense distribution of complex-continuum states.

The most severe problem and future challenge is that the shell-model dimension increases dramatically for $n>2$ particles moving in a large valence space; this is what we henceforth refer to as the dimensionality problem. Using a technique such as the traditional Lanczos iteration method [25] fails in Gamow shell-model calculations. Dealing with large real symmetric matrices, the Lanczos scheme is a powerful method when one wishes to calculate the states lowest in energy. In Gamow shell-model calculations a large number of complex-continuum states may be lying below the physical resonances in real energy. In addition, it is difficult to predict where the multiparticle resonances will appear after diagonalization. In Refs. [6,7], this problem was circumvented by choosing a small number of complex-continuum orbits in the single-particle basis, five points for the ${ }^{6-9} \mathrm{He}$ isotopes.
In such a restricted space of continuum single-particle orbits the authors needed to account for all possible many-particle couplings with continuum states in order to obtain reasonable results for heavier He isotopes. Moreover, the results obtained were not converged with respect to the number of singleparticle continuum orbits. The results reported in Ref. [2] for the He isotopes used fifteen points along the contour. To make calculations feasible for the heavier He isotopes, no more than two particles where allowed to move in complexcontinuum orbitals, invoking the lesson from Refs. [6,7] that configurations with more than two particles in the continuum are of lesser importance. In Ref. [1], this assumption was further justified in Gamow shell-model calculations of the $\mathrm{Li}$ chain. Using an optimal single-particle Hartree-Fock basis, it was found in the ${ }^{7} \mathrm{Li}$ case with three valence particles that the pure continuum configuration has a considerably smaller amplitude than the remaining amplitudes of other configurations.

As pointed out in Refs. [6,7] and expanded on in this work, accurate calculations of multiparticle resonances should in general take into account the effect of all kinds of configurations where particles may move in complex-continuum orbitals, and correspondingly quite a large number of singleparticle complex-continuum orbitals have to be included to obtain satisfactorily converged results. Our aim in this work is to propose an effective interaction scheme to this end. As a first step, we apply the Lee-Suzuki similarity transformation method [26-29] to Gamow shell-model calculations of the ${ }^{6} \mathrm{He}$ and ${ }^{7} \mathrm{He}$ complex energy spectra. The effective interaction is constructed at the two-body level in an optimal single-particle Berggren basis; it is shown that in the ${ }^{7} \mathrm{He}$ case, convergence is much faster using the effective interaction than the bare interaction. Further, since our choice of contour separates the pole configurations from the dense distribution of complexcontinuum states in the unperturbed energy spectrum, we can apply a perturbative treatment to the many-particle resonances, because energy denominators $e_{a}+e_{b}-\left(e_{k}+e_{l}\right)$ appearing in the perturbative expansion are never zero. We apply the non-Hermitian multireference perturbation theory method [30-32] to calculate the ${ }^{7} \mathrm{He}$ spectrum. In defining a suitable model space that contains the most important configurations, we find that second-order corrections are sufficient to give converged energies, using a rather small model space. Finally, we propose a perturbation-theory-based scheme which combines the Lee-Suzuki similarity transformation method and the multireference perturbation method to account for couplings with configurations where all single particles move in complex-continuum orbits. We find that the dimensionality of the model space is drastically smaller than that of the full space, at the converged level. This scheme, tested for three valence particles, may then serve as a starting point for Gamow shell-model calculations of heavier nuclei.

Up to now, Gamow shell-model calculations have been performed with phenomenological nucleon-nucleon interactions. A major challenge is to construct effective nucleon-nucleon interactions for drip-line nuclei starting from a realistic nucleon-nucleon interaction. In this paper, we focus on the choice of contour and the dimensionality problem. The effective nucleon-nucleon interaction adopted is purely 
phenomenological. However, the scheme we present, although implemented with a phenomenological nucleon-nucleon interaction, allows us to define effective interactions computed with the complex scaled single-particle basis. The problem of constructing an effective interaction based on present interaction models for the nucleon-nucleon force will be considered in a forthcoming work.

The outline of this work is as follows. Section II gives a brief description of the contour deformation method in momentum space and presents calculations of the energy spectrum of the nuclei ${ }^{5,6,7} \mathrm{He}$. Section III presents first the Lee-Suzuki transformation method generalized to complex interactions. Thereafter, we apply the similarity transformation method to the unbound nucleus ${ }^{7} \mathrm{He}$ and give a convergence study of the $J^{\pi}=3 / 2_{1}^{-}$resonance, the ground state of ${ }^{7} \mathrm{He}$. Section IV briefly outlines the multireference perturbation method and its application to this state. In Sec. V, we present an effective interaction scheme, which combines the Lee-Suzuki similarity transformation and the multireference perturbation method, for calculation of multiparticle resonances in weakly bound nuclei. Section VI contains an application of our truncation method to the ${ }^{7} \mathrm{He}$ case. Finally, Sec. VII gives the conclusions of the present study and future perspectives and challenges for Gamow shell-model calculations.

\section{THE GAMOW SHELL MODEL}

The newly developed Gamow shell model promises to become a powerful tool in describing and understanding multiparticle resonances appearing in nuclei near the drip lines. Here we discuss how two- and three-particle resonances are formed in ${ }^{6} \mathrm{He}$ and ${ }^{7} \mathrm{He}$, and how they are to be understood in terms of a single-particle picture. Our choice of contour in the complex $k$ plane makes it easy to identify and interpret the multiparticle resonances. In this section, no truncations are made, and all possible configurations within a model space are used in the shell-model calculations.

\section{A. Berggren basis in the momentum representation}

In Ref. [19], we studied the contour deformation method applied to the momentum space Schrödinger equation, and showed how the specific choices of contours based on the analytic structure of the potential may allow the unified description of bound, antibound (virtual), and resonant states. We will apply this method to obtain a single-particle Berggren basis for use in Gamow shell-model calculations. Here we briefly outline the contour deformation method and refer the reader to Ref. [19] for a more rigorous discussion.

The analytically continued single-particle Schrödinger equation on a general inversion symmetric contour takes the form

$$
\frac{\hbar^{2}}{2 \mu} k^{2} \psi_{n l}(k)+\int_{C^{+}} d q q^{2} V_{l}(k, q) \psi_{n l}(q)=E_{n l} \psi_{n l}(k) .
$$

Here both $k$ and $q$ are defined on an inversion symmetric contour $\mathrm{C}^{+}$in the lower half complex $k$ plane, resulting in a closed integral equation. The eigenfunctions constitute a complete biorthogonal set, normalized according to the

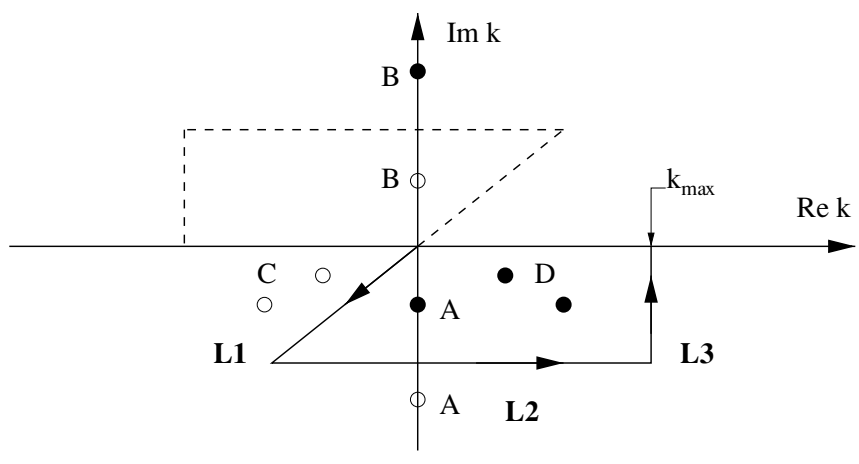

FIG. 1. Contour $C_{R+T}^{+}=L_{1}+L_{2}+L_{3}$ is given by the solid line, while the contour $C_{R+T}^{-}$is given by the dashed line. The contour $C_{R+T}=C_{R+T}^{+}+C_{R+T}^{-}$is inversion symmetric. The single-particle spectrum exposed by this contour is marked by filled circles $\bullet$ and the excluded spectrum by open circles $\circ$. The full spectrum includes bound (B), antibound (A), decay (D), and capture (C) resonant states.

Berggren metric [14-19], namely

$$
\mathbf{1}=\sum_{n \in \mathbf{C}}\left|\psi_{n l}\right\rangle\left\langle\psi_{n l}^{*}\left|+\int_{C^{+}} d k k^{2}\right| \psi_{l}(k)\right\rangle\left\langle\psi_{l}^{*}(k)\right| .
$$

In this work we construct a single-particle Berggren ensemble on a rotated-plus-translated contour $C_{R+T}$ in the complex $k$ plane, studied in detail in Ref. [19]. The contour $C_{R+T}^{+}$is part of the inversion symmetric contour $C_{R+T}=C_{R+T}^{+}+C_{R+T}^{-}$ displayed in Fig. 1. In solving Eq. (1) numerically, we choose a set of $N$ grid points in $k$ space by some quadrature rule, for example, Gauss-Legendre. The integral is then discretized by $\int d k \rightarrow \sum_{i=1}^{N} w_{i}$. On the chosen grid, Eq. (1) takes the complex symmetric form

$$
\frac{\hbar^{2}}{2 \mu} k_{i}^{2} \psi_{n l}(i)+\sum_{j}^{N} \sqrt{w_{i} w_{j}} k_{i} k_{j} V_{l}\left(k_{i}, k_{j}\right) \psi_{n l}(j)=E_{n l} \psi_{n l}(i) .
$$

Here we have defined $\psi_{n l}(i) \equiv \sqrt{w_{i}} k_{i} \psi_{n l}\left(k_{i}\right)$. The norm integral becomes the discrete sum

$$
\delta_{n, n^{\prime}}=\sum_{i=1}^{N} \psi_{n l}(i) \psi_{n^{\prime} l}(i)=\sum_{i=1}^{N} w_{i} k_{i}^{2} \psi_{n l}\left(k_{i}\right) \psi_{n^{\prime} l}\left(k_{i}\right) .
$$

The Berggren completeness given in Eq. (2) takes the discrete form

$$
\mathbf{1}=\sum_{n}^{N}\left|\psi_{n l}(i)\right\rangle\left\langle\psi_{n l}^{*}(i)\right|=\sum_{n}^{N} \sum_{i=1}^{N} \psi_{n l}(i) \psi_{n l}(i) .
$$

Changing from a continuous to a discrete plane-wave basis makes it transparent that the coordinate wave function is an expansion in a basis of spherical-Bessel functions

$$
\phi_{n l}(r)=\sqrt{\frac{2}{\pi}} \sum_{i=1}^{N} \sqrt{w_{i}} k_{i} j_{l}\left(k_{i} r\right) \psi_{n l}(i),
$$

where $\psi_{n l}(i)$ are the expansion coefficients. Defining the functions

$$
f_{l}\left(k_{i} r\right)=\sqrt{\frac{2}{\pi}} \sqrt{w_{i}} k_{i} j_{l}\left(k_{i} r\right),
$$


and using the discrete representation of the Dirac-delta function

$$
\delta\left(k-k^{\prime}\right) \rightarrow \frac{\delta_{k_{i}, k_{j}}}{\sqrt{w_{i} w_{j}}}
$$

we get the expansion

$$
\phi_{n l}(r)=\sum_{i=1}^{N} \psi_{n l}(i) f_{l}\left(k_{i} r\right),
$$

where it is easily seen that the functions $f_{l}\left(k_{i} r\right)$ are orthogonal for different $k_{i}$ and normalized

$$
\int d r r^{2} f_{l}\left(k_{i} r\right) f_{l}\left(k_{j} r\right)=\delta_{k_{i}, k_{j}},
$$

$\delta_{k_{i}, k_{j}}$ being the Kronecker delta. The complete and discrete set of single-particle orbits defined by this contour will then include the pole states, i.e., antibound, bound, and resonant states, and the discretized complex-continuum states defined on each point on the contour. This basis serves as our starting point for Gamow shell-model calculations.

\section{B. Single-particle spectrum of ${ }^{5} \mathrm{He}$}

The unbound nucleus ${ }^{5} \mathrm{He}$ may be modeled by an inert ${ }^{4} \mathrm{He}$ core with a neutron moving mainly in the resonant spin-orbit partners $p_{3 / 2}$ and $p_{1 / 2}$. The $J^{\pi}=3 / 2_{1}^{-}$resonance, to be associated with the single-particle orbit $p_{3 / 2}$, is experimentally known to have a width of $\Gamma \approx 0.60 \mathrm{MeV}$, while the $J^{\pi}=1 / 2_{1}^{-}$ resonance, associated with the single-particle orbit $p_{1 / 2}$, has a large width $\Gamma \approx 4 \mathrm{MeV}$. For more information on these systems, see, for example, the recent review by Jonson [22]. The core-neutron interaction in ${ }^{5} \mathrm{He}$ may be phenomenologically modeled by the SBB (Sack, Biedenharn, and Breit) potential [33]. The SBB potential is of Gaussian type with a spin-orbit term, fitted to reproduce the neutron- ${ }^{4} \mathrm{He}$ scattering phase shifts. In momentum space, the SBB potential, which consists of a central part $c$ and a spin-orbit term $\vec{\sigma} \cdot \vec{l}$, reads

$$
V_{l j}\left(k, k^{\prime}\right)=V_{l j}^{c}\left(k, k^{\prime}\right)+(\vec{\sigma} \cdot \vec{l}) V_{l j}^{\sigma l}\left(k, k^{\prime}\right),
$$

with

$$
\begin{aligned}
V_{l j}^{i}\left(k, k^{\prime}\right)= & -g_{i} \frac{\pi}{4} \frac{a_{i}^{2}}{\sqrt{k k^{\prime}}} \exp \left(\frac{-a_{i}^{2}}{4}\left(k^{2}+k^{\prime 2}\right)\right) \\
& \times I_{l+1 / 2}\left(\frac{a_{i}^{2}}{2} k k^{\prime}\right),
\end{aligned}
$$

where the subscripts $l j$ refer to the single-particle orbital and angular momentum quantum numbers $l$ and $j$, respectively. The term $I_{l+1 / 2}(z)$ is a Bessel function of the first kind with complex arguments. Fitting this potential to reproduce the ${ }^{5} \mathrm{He}$ single-particle resonance spectrum and phase shifts results in $g_{c}=47.4 \mathrm{MeV} g_{\sigma l}=5.86 \mathrm{MeV}$, and $a_{c}=a_{\sigma l}=2.3 \mathrm{fm}$.

In the complex $k$ plane, the Gaussian potential diverges exponentially for $|\operatorname{Im}[k]|>|\operatorname{Re}[k]|$. If we apply the complex scaling technique, which consists of solving the momentum space Schrödinger equation on a purely rotated contour, we get the restriction $\theta<\pi / 4$ on the clockwise rotation angle. Even for smaller angles we may get a poor convergence, since the Gaussian potential oscillates strongly along the rotated

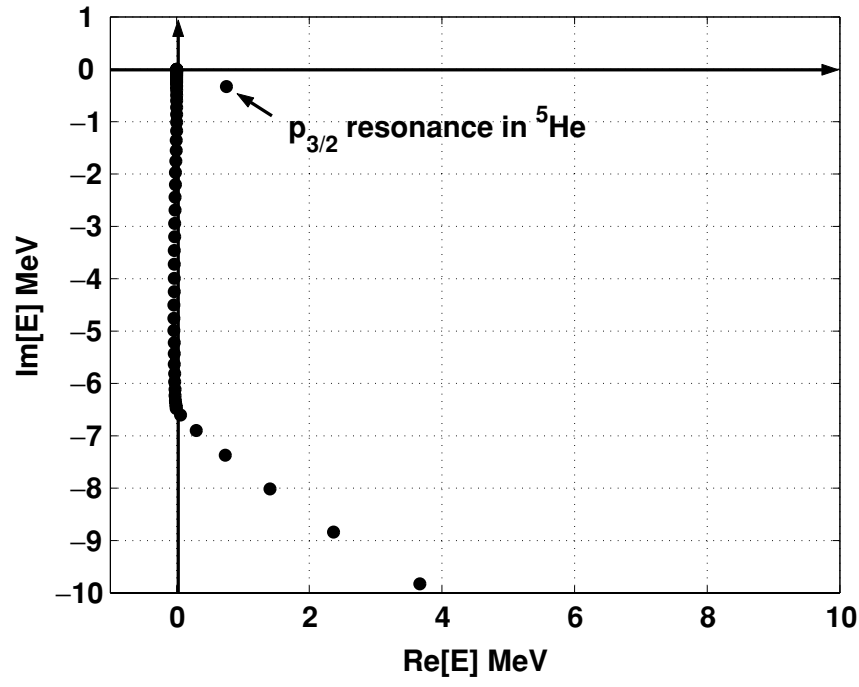

FIG. 2. Plot of the $p_{3 / 2}$ single-particle spectrum in ${ }^{5} \mathrm{He}$ for a Gaussian single-particle potential. The resonance is well located. The remaining points represent the nonresonant continuum.

contours. On the other hand, choosing a contour of the type $C_{R+T}^{+}$solves this problem, allowing for a continuation in the third quadrant of the complex $k$ plane. Furthermore, it yields a faster, smoother decay of the Gaussian potential along the chosen contour.

Since ${ }^{5} \mathrm{He}$ has only resonances in its spectrum, viz., no antibound states, there is no need for an analytic continuation in the third quadrant of the complex $k$ plane, as done in Ref. [19] for the free nucleon-nucleon interaction. We choose a contour of the type $C_{R+T}^{+}$rotated with $\theta=\pi / 4$ and translated with $|\operatorname{Im}[k]|=0.4 \sin (\pi / 4) \approx 0.28 \mathrm{fm}^{-1}$ in the fourth quadrant of the complex $k$ plane. Figures 2 and 3 give plots of the singleparticle spectrum in ${ }^{5} \mathrm{He}$ for the spin-orbit partners $p_{3 / 2}$ and $p_{1 / 2}$, respectively. We have used fifty integration points along both the rotated $C_{R}$ and the translated $C_{T}$ parts of the contour $C_{R+T}^{+}$in the complex $k$ plane.

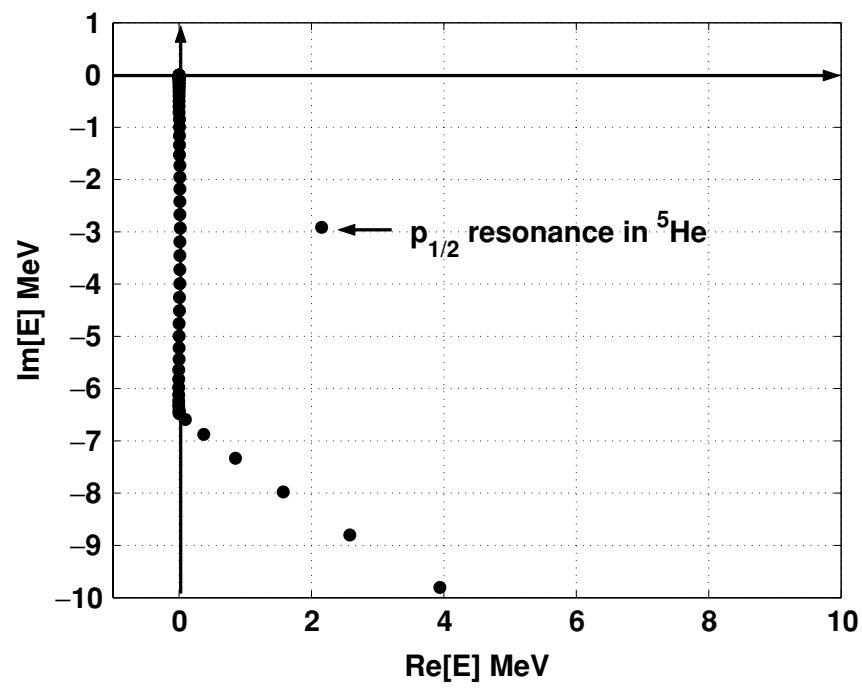

FIG. 3. Same as Fig. 2, but for the $p_{1 / 2}$ single-particle spectrum. 
TABLE I. Convergence of $p_{3 / 2}$ and $p_{1 / 2}$ resonance energies in ${ }^{5} \mathrm{He}$ as function of the number of integration points $N_{R}$ along the rotated $C_{R}$ and $N_{T}$ along the translated part $C_{T}$ of the contour. Energies are in $\mathrm{MeV}$.

\begin{tabular}{ccccccc}
\hline \hline & & \multicolumn{2}{c}{$J^{\pi}=3 / 2^{-}$} & & \multicolumn{2}{c}{$J^{\pi}=1 / 2^{-}$} \\
\cline { 3 - 4 } \cline { 6 - 7 }$N_{R}$ & $N_{T}$ & $\operatorname{Re}[E]$ & $\operatorname{Im}[E]$ & & $\operatorname{Re}[E]$ & $\operatorname{Im}[E]$ \\
\hline 10 & 10 & 0.752321 & -0.329830 & & 2.148476 & -2.912522 \\
12 & 12 & 0.752495 & -0.327963 & & 2.152992 & -2.913609 \\
20 & 20 & 0.752476 & -0.328033 & & 2.154139 & -2.912148 \\
30 & 30 & 0.752476 & -0.328033 & & 2.154147 & -2.912162 \\
40 & 40 & 0.752476 & -0.328033 & & 2.154147 & -2.912162 \\
\hline \hline
\end{tabular}

Table I gives the convergence of the $p_{3 / 2}$ and the $p_{1 / 2}$ singleparticle resonances as a function of integration points along the contour $C_{R+T}$. We observe that already with twelve points along the rotated path and twelve points along the translated line, one has a reasonable convergence of the resonance energy, giving in total forty-eight single-particle states for the valence space consisting of the $l j$ orbits $\left\{p_{3 / 2}, p_{1 / 2}\right\}$ with their pertinent momenta $k$ defined by the number of mesh points. If several particles were to move in this space, clearly the dimensionality would become enormous. It is therefore important, even at the single-particle stage, to optimize the distribution of continuum orbits, so that the main features of the system are reproduced with a small number of singleparticle resonances and complex-continuum orbits. Notice also that the calculated width of the $1 / 2^{-}$resonance is somewhat larger $(\approx 6 \mathrm{MeV})$ than the experimental value $(\approx 4 \mathrm{MeV})[22]$.

\section{Two-particle resonances in ${ }^{6} \mathrm{He}$, including $p_{3 / 2}$ and $p_{1 / 2}$ orbits}

Next we present results for the resonant spectra of ${ }^{6} \mathrm{He}$. We employ again a shell-model picture with ${ }^{6} \mathrm{He}$ modeled by an inert ${ }^{4} \mathrm{He}$ core and two valence neutrons moving in the $l j$ orbits $\left\{p_{3 / 2}, p_{1 / 2}\right\}$, ignoring the recoil of the core. The model space consists then of all momenta $k$ defined by the set of mesh points along the various contours pertinent to these two $l j$ orbits. Using the single-particle wave functions for ${ }^{5} \mathrm{He}$ of Sec. II B, we can in turn construct an antisymmetric two-body wave function based on these single-particle wave functions, viz.,

$$
\Psi_{\alpha}^{J M}(1,2)=\sum_{a \leqslant b} C_{a, b}^{J M} \Phi_{a, b}^{J M}(1,2),
$$

where the indices $a, b$ represent the various single-particle orbits. Here $\Phi_{a, b}^{J}(1,2)$ is an antisymmetric two-particle basis state in the $j-j$ coupling scheme. The sum over single-particle orbits is limited by $a \leqslant b$ since we deal only with identical particles. The expansion coefficients fulfill the completeness relation

$$
1=\sum_{a \leqslant b}\left(C_{a, b}^{J M}\right)^{2}
$$

and the two-particle Berggren basis forms a complete set

$$
1=\sum_{a \leqslant b}\left|\Phi_{a, b}^{J M}(1,2)\right\rangle\left\langle\tilde{\Phi}_{a, b}^{J M}(1,2)\right| .
$$

Here $\left\langle\tilde{\Phi}_{a, b}^{J M}(1,2)\right|$ is the complex conjugate of $\left\langle\Phi_{a, b}^{J M}(1,2)\right|$. We use an effective two-neutron interaction $V_{i j}$, a phenomenological interaction of Gaussian type, separable in $\mathbf{r}_{i}, \mathbf{r}_{j}$ and with interaction strength $V_{0}$, given by

$$
V_{i j}\left(\mathbf{r}_{i}, \mathbf{r}_{j}\right)=V_{0} G\left(r_{i}, r_{j} ; a\right) \sum_{\lambda}\left(Y_{\lambda}(i) \cdot Y_{\lambda}(j)\right),
$$

where

$$
G\left(r_{i}, r_{j} ; a\right)=\exp \left(-\frac{\left(r_{i}^{2}+r_{j}^{2}\right)}{a^{2}}\right) .
$$

The matrix elements of Eq. (16) become

$$
\left\langle\tilde{\Phi}_{a, b}^{J M}(1,2)\left|V_{12}\right| \Phi_{c, d}^{J M}(1,2)\right\rangle=V_{0} \frac{\left\langle\tilde{\Gamma}_{a, b}^{J M}(1,2)\left|V_{12}\right| \Gamma_{c, d}^{J M}(1,2)\right\rangle+(-1)^{J-j_{c}-j_{d}+T}\left\langle\tilde{\Gamma}_{a, b}^{J M}(1,2)\left|V_{12}\right| \Gamma_{d, c}^{J M}(1,2)\right\rangle}{\sqrt{\left(1+\delta_{a b}\right)\left(1+\delta_{c d}\right)}}
$$

Here $\Gamma$ labels nonantisymmetrized two-particle states. Further, the nonantisymmetrized direct matrix elements are given by

$$
\begin{aligned}
\left\langle\tilde{\Gamma}_{a, b}^{J M}(1,2)\left|V_{12}\right| \Gamma_{c, d}^{J M}(1,2)\right\rangle=\frac{1}{2}(-1)^{j_{a}+j_{c}+J} \hat{j}_{a} \hat{j_{b}} \hat{j}_{c} \hat{j}_{d} \\
\quad \times F(a, b ; c, d) \sum_{\lambda}\left\{1+(-1)^{l_{a}+l_{c}+\lambda}\right\}\left\{\begin{array}{ccc}
j_{a} & j_{b} & J \\
j_{d} & j_{c} & \lambda
\end{array}\right\} \\
\quad \times\left(\begin{array}{cccc}
j_{a} & \lambda & j_{c} \\
1 / 2 & 0 & -1 / 2
\end{array}\right)\left(\begin{array}{ccc}
j_{b} & \lambda & j_{d} \\
1 / 2 & 0 & -1 / 2
\end{array}\right) .
\end{aligned}
$$

Here $\hat{j}=\sqrt{2 j+1}$ and

$$
\begin{aligned}
F(a, b ; c, d)= & \int d r_{1} r_{1}^{2} \int d r_{2} r_{2}^{2} \phi_{a}\left(r_{1}\right) \phi_{b}\left(r_{2}\right) \\
& \times G\left(r_{1}, r_{2} ; a\right) \phi_{c}\left(r_{1}\right) \phi_{d}\left(r_{2}\right) .
\end{aligned}
$$

By using the expansion of the radial wave functions in spherical Bessel functions, see Eq. (6), we obtained the interaction in the momentum space Berggren basis. Furthermore, the Fourier-Bessel transform of a Gaussian interaction has an analytic form in momentum space, see Eq. (12).

Two model spaces are considered. The first case includes only the $p_{3 / 2}$ single-particle orbit for various values of the momentum $k$ to be defined below. The second model space also includes the $p_{1 / 2}$ single-particle orbit and its relevant momenta. For both model spaces, we fit the interaction strength to reproduce the $0^{+}$binding energy in ${ }^{6} \mathrm{He}$. We have observed that the position of the $2^{+}$resonance in ${ }^{6} \mathrm{He}$ depends on the range $a$ of the Gaussian interaction, even though we fit the strength so that the $0^{+}$ground state does not change with $a$. 
TABLE II. Dependence of the excited $2^{+}$state on the Gaussian range $a$. In all cases the strength $V_{0}$ has been fitted to reproduce the $0^{+}$ binding energy of ${ }^{6} \mathrm{He}$. Only $p_{3 / 2}$ single-particle orbits are included. The Gaussian range $a$ is in fm, the interaction strength $V_{0}$ in $\mathrm{MeV}$, and energy in $\mathrm{MeV}$.

\begin{tabular}{lcccccc}
\hline \hline & & \multicolumn{2}{c}{$J^{\pi}=0^{+}$} & & \multicolumn{2}{c}{$J^{\pi}=2^{+}$} \\
$V_{0}$ & $a$ & $\operatorname{Re}[E]$ & $\operatorname{Im}[E]$ & & $\operatorname{Re}[E]$ & $\operatorname{Im}[E]$ \\
\hline-72.684 & 1.8 & -0.980355 & 0.000047 & & 1.415355 & -0.457567 \\
-17.239 & 2.8 & -0.980205 & 0.000320 & & 1.333732 & -0.355636 \\
-8.213 & 3.8 & -0.980128 & -0.000826 & & 1.271209 & -0.301898 \\
-5.315 & 4.8 & -0.980067 & -0.000759 & & 1.215956 & -0.267521 \\
-4.011 & 5.8 & -0.980394 & -0.022025 & & 1.165703 & -0.246398 \\
-3.3 & 6.8 & -0.980832 & -0.057382 & & 1.038434 & -0.173482 \\
\hline \hline
\end{tabular}

Unfortunately, for larger values of $a$, the energy fit is better, but the convergence as a function of mesh points is poorer. Table II gives the $0^{+}$ground and $2^{+}$excited state for different values of the Gaussian range $a$. For each value of $a$, the strength of the interaction is fitted to reproduce the ground state energy $E\left(J^{\pi}=0^{+}\right) \approx-0.98 \mathrm{MeV}$. The calculations used twelve integration points along the rotated part and twelve points along the translated part of the contour in all cases. The shape of the contour is also the same in all cases considered in Table II and was specified in the previous section. First of all, we observe that for larger values of the Gaussian range $a$, the convergence gets systematically poorer, since a small spurious width appears in the ground-state energy; this small imaginary part of the $0^{+}$binding energy in ${ }^{6} \mathrm{He}$ will eventually disappear if the number of integration points is increased. Secondly, and more importantly, the larger the range $a$ becomes, the more the $2^{+}$resonant energy agrees with the experimentally measured values. This demonstrates that the two-particle resonant spectrum depends strongly on the radial shape of the interaction and suggests that we should rather deal with an effective interaction derived from realistic models for the nucleon-nucleon interaction. In our calculations, we chose a value of $a$ which is a compromise between a small number of mesh points along the contour and a reasonably good fit of the resonant energy spectra.

The parameters used in our calculations are $V_{0}=$ $-5.315 \mathrm{MeV}$ for the model space involving only the $p_{3 / 2}$ states and $V_{0}=-4.549 \mathrm{MeV}$ for a model space consisting of both single-particle quantum states $p_{3 / 2}$ and $p_{1 / 2}$. We use $a=4.8 \mathrm{fm}$ for both model spaces.

Figures 4 and 5 show the $0^{+}$and $2^{+}$energy spectra, respectively, for ${ }^{6} \mathrm{He}$ after a full diagonalization of the twoparticle shell-model equation. The model space is extended to both $p_{3 / 2}$ and $p_{1 / 2}$ single-particle orbits. This model space yields a bound $0_{1}^{+}$state as well as a resonant $0_{2}^{+}$state. Moreover, we obtain two resonant $2^{+}$states. Observe that the choice of contour, $\left(C_{R+T}^{+}\right)$separates all physical relevant states from the dense distribution of complex-continuum orbits in the energy plane. By this choice of contour, the identification of multiparticle resonances is fairly easy, and one may study the resonant trajectories as the interaction strength is varied.

The stability of the $0^{+}$and $2^{+}$results as functions of the number of mesh points is demonstrated in Tables III and IV.

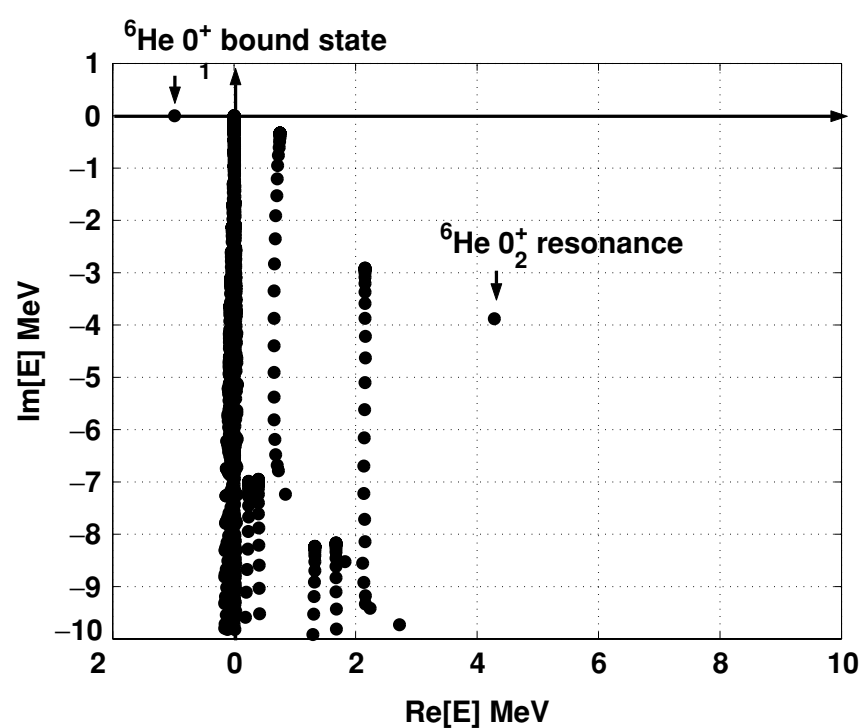

FIG. 4. Plot of the $0_{1}^{+}$bound and $0_{2}^{+}$resonant states in ${ }^{6} \mathrm{He}$ for a model space consisting of the $p_{3 / 2}$ and $p_{1 / 2}$ single-particle orbits. The bound and resonant states are well located. The remaining points represent the nonresonant continuum.

Limiting attention first to a model space consisting only of the $p_{3 / 2}$ orbit, we note that with $N_{R}=12$ integration points along the rotated path $C_{R}$ and $N_{T}=12$ points along the translated line $C_{T}$, convergence is satisfactory, i.e., even with a total of only 300 two-particle states.

Tables V, VI, and VII repeat the above convergence analysis, but they employ the extended model space consisting of the $p_{3 / 2}$ and $p_{1 / 2}$ single-particle orbits and include the results for the lowest-lying ${ }^{6} \mathrm{He}$ state with quantum numbers $J^{\pi}=1^{+}$. Increasing the model space brings several new features. We note in Table $\mathrm{V}$ that the first excited $0_{2}^{+}$state is a resonance. The stability of the results as functions of the number of mesh points is comparable to that seen in Tables III

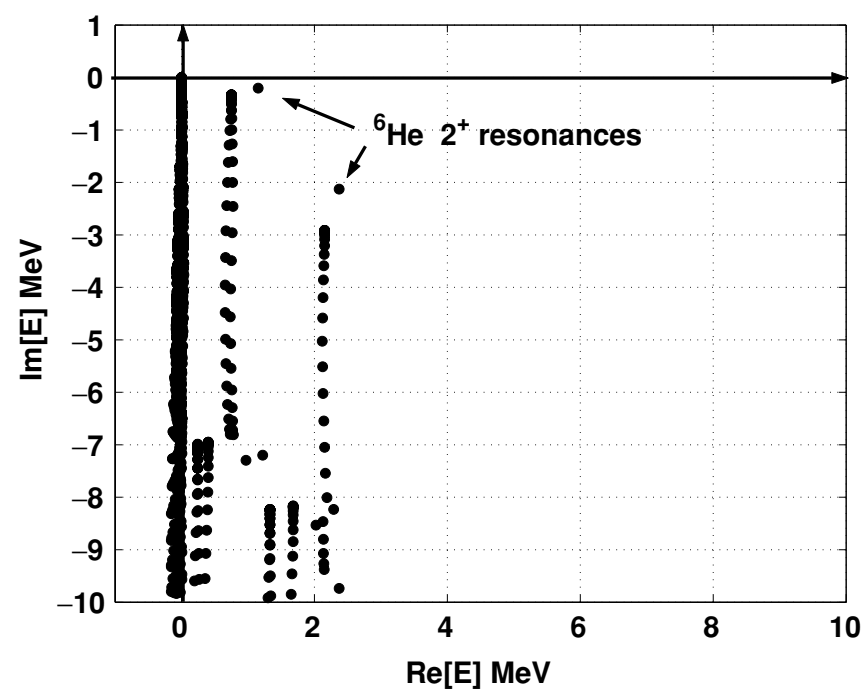

FIG. 5. Plot of $2^{+}$resonances in ${ }^{6} \mathrm{He}$ for a model space consisting of the $p_{3 / 2}$ and $p_{1 / 2}$ single-particle orbits. Both resonant states are well located. The remaining points represent the nonresonant continuum. 
TABLE III. Convergence of the $0_{1}^{+}$bound state energy in ${ }^{6} \mathrm{He}$ in terms of the number of integration points $N_{R}$ and $N_{T}$ along the rotated $C_{R}$ and the translated part $C_{T}$ of the contour, respectively. The number $N_{2 p}$ gives the dimension of the two-particle antisymmetrized basis. Only $p_{3 / 2}$ single-particle orbits are included. Energies are in $\mathrm{MeV}$.

\begin{tabular}{lcrcr}
\hline \hline$N_{R}$ & $N_{T}$ & $N_{2 p}$ & $\operatorname{Re}[E]$ & \multicolumn{1}{c}{$\operatorname{Im}[E]$} \\
\hline 12 & 12 & 300 & -0.980067 & -0.000759 \\
20 & 20 & 820 & -0.979508 & 0.000000 \\
25 & 25 & 1275 & -0.979509 & 0.000000 \\
\hline \hline
\end{tabular}

TABLE IV. Same as Table III, but for the $2_{1}^{+}$resonant state energy.

\begin{tabular}{lcrcc}
\hline \hline$N_{R}$ & $N_{T}$ & $N_{2 p}$ & $\operatorname{Re}[E]$ & $\operatorname{Im}[E]$ \\
\hline 12 & 12 & 300 & 1.215956 & -0.267521 \\
20 & 20 & 820 & 1.216495 & -0.267745 \\
25 & 25 & 1275 & 1.216496 & -0.267745 \\
\hline \hline
\end{tabular}

TABLE V. Same as Table III, but for the $0_{1}^{+}$bound and the $0_{2}^{+}$ resonant state energies. Both the $p_{3 / 2}$ and $p_{1 / 2}$ single-particle orbits are included in $\mathrm{MeV}$.

\begin{tabular}{rrrrrrrr}
\hline \hline & & & \multicolumn{2}{c}{$J^{\pi}=0_{1}^{+}$} & & \multicolumn{2}{c}{$J^{\pi}=0_{2}^{+}$} \\
\cline { 8 - 8 } \cline { 7 - 7 }$N_{R}$ & $N_{T}$ & $N_{2 p}$ & $\operatorname{Re}[E]$ & $\operatorname{Im}[E]$ & & $\operatorname{Re}[E]$ & $\operatorname{Im}[E]$ \\
\hline 12 & 12 & 600 & -0.980111 & -0.000497 & & 4.289194 & -3.882119 \\
20 & 20 & 1640 & -0.979148 & -0.000000 & & 4.286186 & -3.882878 \\
25 & 25 & 2550 & -0.979148 & 0.000000 & & 4.286181 & -3.882876 \\
\hline \hline
\end{tabular}

TABLE VI. Same as Table V, but for the $1_{1}^{+}$resonance.

\begin{tabular}{lcccc}
\hline \hline & & \multicolumn{2}{c}{$J^{\pi}=1^{+}$} \\
\cline { 4 - 5 }$N_{R}$ & $N_{T}$ & $N_{2 p}$ & $\operatorname{Re}[E]$ & $\operatorname{Im}[E]$ \\
\hline 12 & 12 & 1128 & 1.945539 & -2.920286 \\
20 & 20 & 3160 & 1.940263 & -2.930619 \\
25 & 25 & 4950 & 1.940266 & -2.930608 \\
\hline \hline
\end{tabular}

TABLE VII. Same as table V, but for the $2_{1}^{+}$and $2_{2}^{+}$resonance energies.

\begin{tabular}{|c|c|c|c|c|c|c|}
\hline \multirow[b]{2}{*}{$N_{R}$} & \multirow[b]{2}{*}{$N_{T}$} & \multirow[b]{2}{*}{$N_{2 p}$} & \multicolumn{2}{|c|}{$J^{\pi}=2_{1}^{+}$} & \multicolumn{2}{|c|}{$J^{\pi}=2_{2}^{+}$} \\
\hline & & & $\operatorname{Re}[E]$ & $\operatorname{Im}[E]$ & $\operatorname{Re}[E]$ & $\operatorname{Im}[E]$ \\
\hline 12 & 12 & 876 & 1.149842 & -0.203052 & 2.372295 & -2.122474 \\
\hline 20 & 20 & 2420 & 1.150527 & -0.203060 & 2.372818 & -2.123253 \\
\hline 25 & 25 & 3775 & 1.150527 & -0.203060 & 2.372817 & -2.123254 \\
\hline
\end{tabular}

TABLE VIII. Expansion coefficients of the $0_{1}^{+}$bound state in ${ }^{6} \mathrm{He}$. The $p_{3 / 2}$ and $p_{1 / 2}$ single-particle orbits define the model space. See text for further discussions.

\begin{tabular}{lrrrrr}
\hline \hline & \multicolumn{2}{c}{$p_{3 / 2}^{2}$} & & \multicolumn{2}{c}{$p_{1 / 2}^{2}$} \\
\cline { 2 - 3 } \cline { 5 - 6 } & $\operatorname{Re}\left[C^{2}\right]$ & $\operatorname{Im}\left[C^{2}\right]$ & & $\operatorname{Re}\left[C^{2}\right]$ & $\operatorname{Im}\left[C^{2}\right]$ \\
\hline$|R R\rangle$ & 1.10488 & -0.83161 & & 0.22620 & -0.16120 \\
$|R C\rangle$ & -0.06036 & 0.88137 & & -0.19842 & 0.22423 \\
$|C C\rangle$ & -0.09716 & -0.04974 & & 0.02486 & -0.06305 \\
\hline \hline
\end{tabular}

and IV. With approximately twelve mesh points, we obtain results close to the converged ones. Similar conclusions apply to the $1_{1}^{+}$resonance and the two lowest-lying $2^{+}$resonant states; see Tables VI and VII for more details. We note that the experimental value for the width of the first excited $J^{\pi}=2_{1}^{+}$ is $\Gamma \approx 113 \mathrm{keV}$ and the energy is $\operatorname{Re}[E]_{2_{1}^{+}}=1797 \mathrm{keV}$. Our simplified nucleon-nucleon interaction gives a qualitative reproduction of the data. In a future work we plan to include a realistic nucleon-nucleon interaction for studies of such systems.

We end this section by analyzing the squared amplitude of the single-particle configurations $|R R\rangle,|R C\rangle,|C C\rangle$ of the $0^{+}, 1^{+}$, and $2^{+}$bound and resonant wave functions. The results are given in Tables VIII-XII. The reason for doing this analysis is that our single-particle basis consists of both resonant and continuum single-particle orbits. By performing such an analysis we can disentangle the contribution from, for example, the nonresonant continuum. In these tables, $|R R\rangle$ stands for both single-particle orbits being in a resonant single-particle orbit, $|R C\rangle$ means that one single-particle orbit is a resonant single-particle orbit and the other a nonresonant continuum single-particle orbit, while for $|C C\rangle$ both single-particle orbits are from the nonresonant single-particle continuum. All the results show that the configurations where both single particles are in resonant orbits have the largest amplitude in the two-body wave function. It is also seen that the configurations where both particles are in complex-continuum orbits have a small effect on the formation of two-particle resonances in ${ }^{6} \mathrm{He}$. This is a useful result which we will exploit below when we define effective interactions for smaller spaces.

\section{Three-particle resonances in ${ }^{7} \mathrm{He}$, including $p_{3 / 2}$ only}

Finally we consider the unbound nucleus ${ }^{7} \mathrm{He}$, with a ground state $\left(J^{\pi}=3 / 2^{-}\right)$located $\approx 0.5 \mathrm{MeV}$ above the ${ }^{6} \mathrm{He}$ ground state and with a measured width $\Gamma \approx 160 \mathrm{keV}$. Other continuum structures, with tentative spin assignments

TABLE IX. Same as Table VIII, but for the $0_{2}^{+}$resonance.

\begin{tabular}{lrrrrr}
\hline \hline & \multicolumn{2}{c}{$p_{3 / 2}^{2}$} & & \multicolumn{2}{c}{$p_{1 / 2}^{2}$} \\
\cline { 2 - 3 } \cline { 5 - 6 } & $\operatorname{Re}\left[C^{2}\right]$ & $\operatorname{Im}\left[C^{2}\right]$ & & $\operatorname{Re}\left[C^{2}\right]$ & $\operatorname{Im}\left[C^{2}\right]$ \\
\hline$|R R\rangle$ & -0.01136 & -0.08003 & & 0.90189 & 0.33029 \\
$|R C\rangle$ & 0.04282 & -0.03939 & & 0.05966 & -0.24478 \\
$|C C\rangle$ & 0.00617 & 0.00494 & & 0.00082 & 0.02896 \\
\hline \hline
\end{tabular}


TABLE X. Same as Table VIII, but for the $1^{+}$resonance.

\begin{tabular}{|c|c|c|c|c|c|c|}
\hline & \multicolumn{2}{|c|}{$p_{1 / 2} p_{3 / 2}$} & \multicolumn{2}{|c|}{$p_{1 / 2}^{2}$} & \multicolumn{2}{|c|}{$p_{3 / 2}^{2}$} \\
\hline & $\operatorname{Re}\left[C^{2}\right]$ & $\operatorname{Im}\left[C^{2}\right]$ & $\operatorname{Re}\left[C^{2}\right]$ & $\operatorname{Im}\left[C^{2}\right]$ & $\operatorname{Re}\left[C^{2}\right]$ & $\operatorname{Im}\left[C^{2}\right]$ \\
\hline$|R R\rangle$ & 0.71068 & -0.03739 & & & & \\
\hline$|R C\rangle$ & 0.00381 & -0.06948 & 0.00016 & 0.00003 & 0.02224 & 0.01171 \\
\hline$|C R\rangle$ & 0.20647 & 0.05208 & 0.00037 & 0.00071 & 0.07067 & 0.03807 \\
\hline$|C C\rangle$ & -0.00984 & -0.00149 & -0.00031 & -0.00009 & -0.00424 & 0.00585 \\
\hline
\end{tabular}

$J^{\pi}=1 / 2^{-}$, and $5 / 2^{-}$, have been observed; see, for example, Ref. [22] for an extensive review of the experimental situation. In this section we limit our attention to a model defined only by the $p_{3 / 2}$ single-particle orbits, and we consider only the $J^{\pi}=3 / 2^{-}$resonance. The reason we do not include the $p_{1 / 2}$ single-particle orbits is that we aim at obtaining a diagonalization in the full space, taking into account all complex-continuum couplings. This model calculation will serve later as a reference. In the case of 24 mesh points in momentum space for the $p_{3 / 2}$ single-particle quantum numbers $l j$, the total dimension $d$ of the $\left(J^{\pi}=3 / 2^{-}\right)$three-particle problem is $d=9224$. If, in addition, we were to include 24 single-particle momenta for the $p_{1 / 2}$ single-particle quantum numbers $l j$, we would have roughly $d \sim 40000$ three-body configurations. We will return to the full $p_{3 / 2}, p_{1 / 2}$ space in Sec. VI, using the truncation scheme of the following sections. In Refs. [6] and [7], the dimensionality problem was circumvented by choosing a small number of complexcontinuum orbits, typically about five, although it was found that a larger number of continuum orbits had to be included to obtain converged results. As for ${ }^{6} \mathrm{He}$, we construct a three-body wave function using the single-particle wave functions defined in ${ }^{5} \mathrm{He}$. The three-body wave function is expanded in a three-particle antisymmetric Berggren basis

$$
\Psi_{\alpha}^{J M}(1,2,3)=\sum_{a \leqslant b \leqslant c} C_{(a, b) c}^{J M} \Phi_{(a, b) c}^{J M}(1,2,3),
$$

where the completeness relation reads

$$
1=\sum_{a \leqslant b \leqslant c}\left|\Phi_{(a, b) c}^{J M}(1,2,3)\right\rangle\left\langle\tilde{\Phi}_{(a, b) c}^{J M}(1,2,3)\right|,
$$

with

$$
1=\sum_{a \leqslant b \leqslant c}\left(C_{(a, b) c)}^{J M}\right)^{2}
$$

The two-body nucleon-nucleon interaction is the same as that used for ${ }^{6} \mathrm{He}$. Figure 6 gives the energy spectrum after a

TABLE XI. Same as Table VIII, but for the $2_{1}^{+}$resonance.

\begin{tabular}{lrrrrr}
\hline \hline & \multicolumn{2}{c}{$p_{1 / 2} p_{3 / 2}$} & & \multicolumn{2}{c}{$p_{3 / 2}^{2}$} \\
\cline { 2 - 3 } \cline { 5 - 6 } & $\operatorname{Re}\left[C^{2}\right]$ & $\operatorname{Im}\left[C^{2}\right]$ & & $\operatorname{Re}\left[C^{2}\right]$ & $\operatorname{Im}\left[C^{2}\right]$ \\
\hline$|R R\rangle$ & 0.11394 & -0.00494 & & 0.96962 & 0.05539 \\
$|R C\rangle$ & -0.00474 & 0.02531 & & -0.00178 & -0.00018 \\
$|C R\rangle$ & -0.02776 & -0.03796 & & -0.05069 & -0.02708 \\
$|C C\rangle$ & 0.00229 & -0.00772 & & -0.00089 & -0.00282 \\
\hline \hline
\end{tabular}

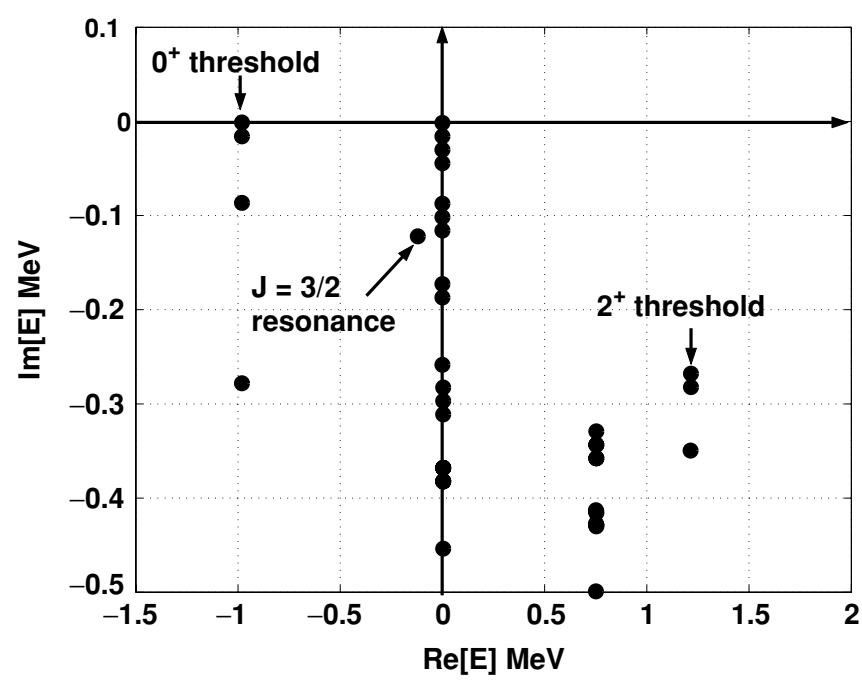

FIG. 6. Plot of the $3 / 2^{-}$complex energy spectrum of ${ }^{7} \mathrm{He}$ for a model space consisting of $p_{3 / 2}$ single-particle orbits only. The $J^{\pi}=3 / 2^{-}$resonance is located at $E_{3 / 2^{-}}=-(0.120731+$ $0.122211 i) \mathrm{MeV}$.

full diagonalization of the three-particle Gamow shell-model equation. It is seen that the choice of contour in calculating the single-particle spectrum is again optimal since all interesting physical states are well separated from the dense distribution of complex scattering states. The $J^{\pi}=3 / 2^{-}$resonance appears at energy $E_{3 / 2^{-}}=-(0.12+0.12 i) \mathrm{MeV}$. The $J^{\pi}=3 / 2^{-}$ energy spectrum of ${ }^{7} \mathrm{He}$ plotted in Fig. 6 shows that the $0^{+}$and $2^{+}$states in ${ }^{6} \mathrm{He}$, and the $3 / 2^{-}$state in ${ }^{5} \mathrm{He}$, form complex thresholds. The physical interpretation of these three-particle states is, in the case of the ${ }^{6} \mathrm{He}$ thresholds, that two of the neutrons form either the $0^{+}$ground state or the $2^{+}$resonant state, while the third neutron is moving in a complex-continuum orbit. In the case of the ${ }^{5} \mathrm{He}$ complex threshold, two neutrons move in complex-continuum orbits while the third forms the $3 / 2^{-}$ground state in ${ }^{5} \mathrm{He}$.

A diagonalization within the reduced space, where at most two particles move in continuum orbits gives the resonance energy $-(0.14+0.16 i) \mathrm{MeV}$, which shows that the effect coming from all particles moving in the continuum is not neglible, but small.

Table XIII gives the squared amplitudes of the various single-particle configurations in the ${ }^{7} \mathrm{He}$ ground state, $\{|R R R\rangle,|R R C\rangle,|R C C\rangle,|C C C\rangle\}$, where again $R$ labels a single-particle resonance and $C$ a complex single-particle continuum orbit. The most important configuration, as in the

TABLE XII. Same as Table VIII, but for the $2_{2}^{+}$resonance.

\begin{tabular}{lrrrrr}
\hline \hline & \multicolumn{2}{c}{$p_{1 / 2} p_{3 / 2}$} & & \multicolumn{2}{c}{$p_{3 / 2}^{2}$} \\
\cline { 2 - 3 } \cline { 5 - 6 } & $\operatorname{Re}\left[C^{2}\right]$ & $\operatorname{Im}\left[C^{2}\right]$ & & $\operatorname{Re}\left[C^{2}\right]$ & $\operatorname{Im}\left[C^{2}\right]$ \\
\hline$|R R\rangle$ & 0.88847 & -0.03742 & & 0.08911 & -0.03742 \\
$|R C\rangle$ & -0.04888 & 0.05674 & & -0.00104 & -0.00055 \\
$|C R\rangle$ & 0.06058 & -0.03336 & & -0.00072 & -0.02469 \\
$|C C\rangle$ & 0.01131 & -0.00447 & & 0.00115 & -0.00220 \\
\hline \hline
\end{tabular}


TABLE XIII. Expansion coefficients of the $J^{\pi}=3 / 2^{-}$ground state in ${ }^{7} \mathrm{He}$. Only $p_{3 / 2}$ single-particle orbits are included.

\begin{tabular}{lcr}
\hline \hline & $\left|p_{3 / 2}^{3}\right\rangle$ & \\
\hline & $\operatorname{Re}\left[C^{2}\right]$ & \multicolumn{1}{c}{$\operatorname{Im}\left[C^{2}\right]$} \\
\hline$|R R R\rangle$ & 1.295549 & -0.986836 \\
$|R R C\rangle$ & -0.184544 & 1.099729 \\
$|R C C\rangle$ & -0.115738 & -0.110375 \\
$|C C C\rangle$ & 0.004733 & -0.002518 \\
\hline \hline
\end{tabular}

case of ${ }^{6} \mathrm{He}$, is the one where all single-particles are in the $p_{3 / 2}$ single-particle resonant orbit. The effect of configurations where all particles are in continuum orbits is small, which suggests that the coupling to configurations $|C C C\rangle$ may be taken into account perturbatively. We will exploit this feature in Secs. III and IV.

In Fig. 6 we note that the $J^{\pi}=3 / 2^{-}$ground state in ${ }^{7} \mathrm{He}$ appears at an energy of approximately $0.86 \mathrm{MeV}$ above the ground state in ${ }^{6} \mathrm{He}$, while the experimental value is at approximately $0.5 \mathrm{MeV}$. This discrepancy with experiment can be understood in terms of the configuration $|R R R\rangle$, and the choice of interaction. Focusing on the first aspect and using coefficients of fractional parentage, we can rewrite the $|R R R\rangle$ configuration as

$$
\begin{aligned}
\left|\left(p_{3 / 2}\right)^{3} ; J^{\pi}=3 / 2^{-}\right\rangle= & \frac{1}{6}\left|\left(p_{3 / 2}\right)_{0}^{2} p_{3 / 2} ; J^{\pi}=3 / 2^{-}\right\rangle-\sqrt{\frac{5}{6}} \\
& \times\left|\left(p_{3 / 2}\right)_{2}^{2} p_{3 / 2} ; J^{\pi}=3 / 2^{-}\right\rangle .
\end{aligned}
$$

From the geometry, one may conclude that the ground state of ${ }^{7} \mathrm{He}$ bears much more resemblance to the $2_{1}^{+}$resonance than to the $0_{1}^{+}$ground state of ${ }^{6} \mathrm{He}$. In our calculations, the $2_{1}^{+}$resonance comes at an energy $\approx(1.2-0.26 i) \mathrm{MeV}$, which is roughly $2.2 \mathrm{MeV}$ above the $0_{1}^{+}$ground state of ${ }^{6} \mathrm{He}$, to be contrasted with the experimental value of $\approx 1.8 \mathrm{MeV}$. This suggests that if we were to increase the attractive strength of the $J^{\pi}=2^{+}$interaction in ${ }^{6} \mathrm{He}$ and achieve a better agreement with the experimental value, the $J^{\pi}=3 / 2^{-}$resonant ground state of ${ }^{7} \mathrm{He}$ would also get closer to the experimental results.

\section{EFFECTIVE INTERACTIONS FOR THE GAMOW SHELL MODEL}

\section{A. Lee-Suzuki similarity transformation for complex interactions}

The previous section introduced and motivated the application of complex scaling and a Berggren basis in studies of weakly bound nuclear systems. However, employing such a momentum space basis soon exceeds feasible dimensionalities in shell-model studies. To circumvent this problem and to be able to define effective interactions of practical use in shell-model calculations, we introduce effective two-body interactions based on similarity transformation methods. These interactions are in turn employed in Gamow shell-model calculations. We base our approach on the extensive works of Suzuki, Okamoto, Lee, and collaborators [26-29]. This similarity transformation method has been widely used in the construction of effective two- and three-body interactions for use in the no-core shell-model approach of Barrett, Navrátil, Vary, and collaborators (see [34-37] and references therein). However, since the similarity transformation method has previously only been considered for real interactions, we need to extend its use to Gamow shell-model calculations, implying a generalization to complex interactions.

To achieve this, we first introduce the two-body Schrödinger equation

$$
H\left|\Psi_{\alpha}^{J}\right\rangle=\left(H_{0}+V_{12}\right)\left|\Psi_{\alpha}^{J}\right\rangle=E_{\alpha}\left|\Psi_{\alpha}^{J}\right\rangle,
$$

which in our specific case represents the ${ }^{6} \mathrm{He}$ shell-model equations discussed in detail in Sec. II C. Here $H_{0}$ includes the single-particle part of the Hamiltonian, kinetic energy, and an eventual single-particle potential. The term $V_{12}$ is the residual two-body interaction, which we have chosen to be of separable Gaussian form, see Eq. (16). The exact wave function $\Psi_{\alpha}^{J}$ is expanded in the antisymmetric two-particle basis of Eq. (13) generated from the single-particle basis of $H_{0}$, corresponding to the basis from the ${ }^{5} \mathrm{He}$ calculations of Sec. II B.

The aim is to construct an effective interaction in a reduced two-particle space (model space). The most natural way of defining the two-particle model space is to start from a singleparticle formulation. Starting with the single-particle Berggren basis for ${ }^{5} \mathrm{He}$, this space is divided into two subspaces, i.e., a single-particle model space $p$ and a corresponding complement space $q$. These single-particle spaces define, in turn, our two(and many-) particle model spaces

$$
P=\sum_{a \leqslant b}\left|\Phi_{a, b}^{J}(1,2)\right\rangle\left\langle\tilde{\Phi}_{a, b}^{J}(1,2)\right|, \quad a, b \in p,
$$

and the complement space

$$
Q=\sum_{a \leqslant b}\left|\Phi_{a, b}^{J}(1,2)\right\rangle\left\langle\tilde{\Phi}_{a, b}^{J}(1,2)\right|, \quad\left\{\begin{array}{c}
a \in p \wedge b \in q, \\
a, b \in q .
\end{array}\right.
$$

The antisymmetric two-particle basis follows the Berggren metric and is normalized according to

$$
\left\langle\tilde{\Phi}_{a, b}^{J}(1,2) \mid \Phi_{c, d}^{J}(1,2)\right\rangle=\frac{\delta_{a, c} \delta_{b, d}-(-1)^{j_{a}+j_{b}-J} \delta_{a, d} \delta_{b, c}}{\sqrt{\left(1+\delta_{a, b}\right)\left(1+\delta_{c, d}\right)}} .
$$

The projection operators fulfill the relations

$$
P^{2}=P, \quad Q^{2}=Q, \quad P^{\mathrm{T}}=P,
$$

and

$$
Q^{\mathrm{T}}=Q, \quad P+Q=1, \quad P Q=0,
$$

where $T$ indicates the transpose. The first challenge is to define a suitable single-particle model space within the Berggren formalism. Ideally the model space should consist of the single-particle orbitals which in the two-, three- and many-body problems give the most important many-body correlations. In defining $p$ within the no-core shell model approach [34-37], one typically selects the $n_{p}$ lowest oscillator states. When dealing with a single-particle Berggren basis, selecting $p$ is not a straightforward procedure. First, it is rather obvious that the single-particle resonant orbitals should be part 
of $p$; on the other hand, it is not obvious which nonresonant continuum orbitals should be part of $p$. For example, should one choose the continuum orbitals lowest in real, imaginary, or absolute value of the energy? Or should one rather choose the nonresonant continuum orbitals closest in energy to the single-particle resonances?

Our prescription of selecting $p$ is based on our knowledge of the physical system, ${ }^{7} \mathrm{He}$, in which we ultimately wish to apply the effective two-body interaction. In Sec. II, we saw that the $J^{\pi}=3 / 2^{-}$ground state of ${ }^{7} \mathrm{He}$ has the $2^{+}$resonance in ${ }^{6} \mathrm{He}$ as an important two-body configuration [see Eq. (24)]. Based on this result, a viable starting point is to study the singleparticle strengths in the $2^{+}$resonance wave function. To understand the nature of two-particle resonances and how they are formed in a shell-model framework, it is natural to study and analyze the single-particle strengths in the two-particle wave function and to determine how they are distributed among the single-particle resonances and the various complex-continuum orbits, given a specific contour in the complex $k$ plane.

The single-particle density operator is given by

$$
\hat{n}_{i}=\sum_{j}^{N}\left|\psi_{i}(j)\right\rangle\left\langle\tilde{\psi}_{i}(j)\right|, \quad N=\sum_{i} \hat{n}_{i},
$$

where $N$ is the total number of particles, and $i$ represents the single-particle orbit and its quantum number. In the case of ${ }^{6} \mathrm{He}$ with an inert ${ }^{4} \mathrm{He}$ core, $N=2$. To find the probability $n_{i}$ that either particle 1 or particle 2 is in the single-particle orbit $i$, we calculate the matrix element of $\hat{n}_{i}$ with the two-particle resonance wave function,

$$
\begin{aligned}
n_{i}= & \left\langle\tilde{\Psi}_{\alpha}^{J}(1,2)\left|\hat{n}_{i}\right| \Psi_{\alpha}^{J}(1,2)\right\rangle \\
= & \sum_{a \leqslant b} \sum_{c \leqslant d} C_{a, b}^{\alpha} C_{c, d}^{\alpha}\left(\frac{1}{\left(1+\delta_{a, b}\right)\left(1+\delta_{c, d}\right)}\right)^{1 / 2} \\
& \times\left\{\delta_{d, i} \delta_{c, i} \delta_{b, d}+(-1)^{j_{c}+j_{i}-J+1} \delta_{a, i} \delta_{d, i} \delta_{b, c}\right. \\
& \left.+\delta_{b, i} \delta_{d, i} \delta_{a, c}+(-1)^{j_{a}+j_{i}-J+1} \delta_{b, i} \delta_{c, i} \delta_{a, d}\right\} .
\end{aligned}
$$

Figure 7 gives the real, imaginary, and absolute values of the single-particle strength among the complex-continuum orbits in the $2^{+}$resonance in ${ }^{6} \mathrm{He}$. The strengths are plotted as a function of the absolute value of the complex-continuum energy. Observe that the continuum states near the $2^{+}$resonance in ${ }^{6} \mathrm{He}$ have the largest strength. This may be understood as an interference effect between the single-particle resonance and the continuum orbits located closest in energy (momentum) to the $p_{3 / 2}$ single-particle resonance. When defining a singleparticle model space, we choose the single-particle resonant and complex-continuum orbits with the largest absolute value of the single-particle strength. With this recipe, we have a consistent way of defining a single-particle model space, which forms the basis for constructing an effective interaction in the two-particle model space.

Having defined a two-particle model space, we now wish to construct an effective two-body interaction within $P$, which reproduces in the $P$ space exactly $N_{P}$ selected eigenvalues of the full Hamiltonian. This can be accomplished by a similarity

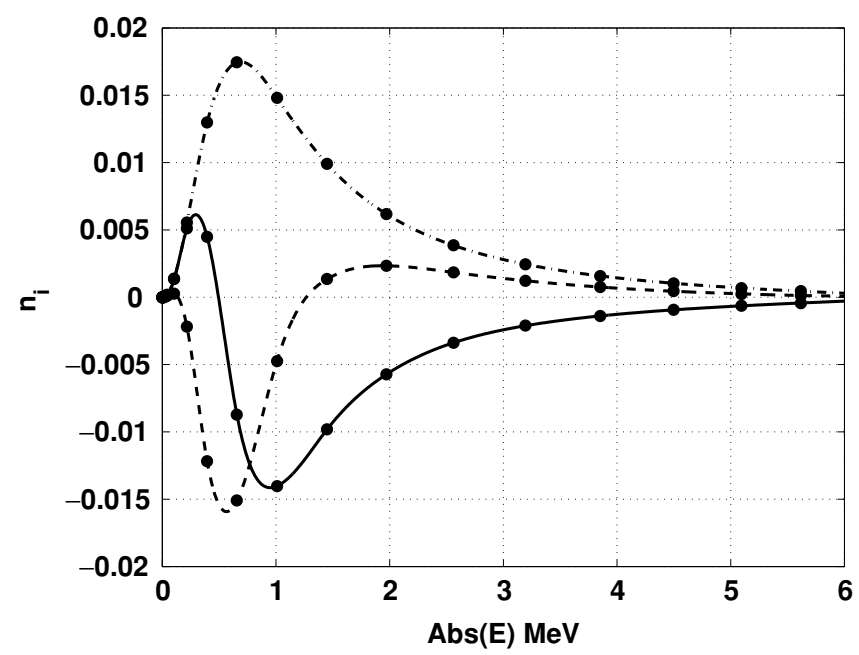

FIG. 7. Plot of the $p_{3 / 2}$ single-particle complex-continuum strength $n_{i}$ in the two-particle resonance wave function $2_{1}^{+}$in ${ }^{6} \mathrm{He}$. The solid line gives the real part, the dashed line the imaginary, and the dash-dotted line the absolute value of the strength. The filled circles give the actual location of the complex-continuum states in the absolute value of energy.

transformation

$$
\tilde{H}=e^{-\omega} H e^{\omega},
$$

where $\omega$ is defined by $\omega=Q \omega P$. It follows that $\omega^{2}=\omega^{3}=$ $\cdots=0$ and $e^{\omega}=P+Q+\omega$. The two-body shell-model equation (25) can then be rewritten in a $2 \times 2$ block structure

$$
\left(\begin{array}{cc}
P \tilde{H} P & P \tilde{H} Q \\
Q \tilde{H} P & Q \tilde{H} Q
\end{array}\right)\left(\begin{array}{c}
P \Psi_{\alpha}^{J} \\
Q \Psi_{\alpha}^{J}
\end{array}\right)=E_{n}\left(\begin{array}{c}
P \Psi_{\alpha}^{J} \\
Q \Psi_{\alpha}^{J}
\end{array}\right) .
$$

If $P \tilde{H} P$ is to be the two-particle effective interaction, reproducing exactly $N_{P}$ eigenvalues of $H$, the decoupling condition $P \tilde{H} Q=0$ must be fulfilled. One may show that the decoupling condition becomes [26,27]

$$
Q H P+Q H Q \omega-\omega P H P-\omega P H Q \omega=0,
$$

with $\omega$ acting as a transformation from the model space $P$ to its complement $Q$, viz.,

$$
\left\langle\tilde{\Phi}_{c, d}^{J} \mid \Psi_{\alpha}^{J}\right\rangle=\sum_{a \leqslant b}\left\langle\tilde{\Phi}_{c, d}^{J}|\omega| \Phi_{a, b}^{J}\right\rangle\left\langle\tilde{\Phi}_{a, b}^{J} \mid \Psi_{\alpha}^{J}\right\rangle,
$$

with $\Phi_{a, b}^{J} \in P$ and $\Phi_{c, d}^{J} \in Q$, respectively. In constructing the two-body effective interaction, one obviously needs the solution for the transformation operator $\omega$. This is obtained in two steps. First, the two-body shell-model equation (25) is solved exactly; this is done for ${ }^{6} \mathrm{He}$ in Sec. II $\mathrm{C}$ for all relevant spins. Second, $N_{P}$ exact solutions of Eq. (25) are selected and entered into Eq. (36). Now the question arises as to which $N_{P}$ exact solutions should be picked. The effective interaction generated in the model space depends on the $N_{P}$ exact solutions entering Eq. (36). This is why the effective interaction generated by the similarity transformation method is often referred to as a state-dependent effective interaction, and there is no unique solution for $\omega$. From Eq. (36), it is seen that the solution for $\omega$ may be obtained as long as the 
matrix $\left\langle\tilde{\Phi}_{a, b}^{J} \mid \Psi_{\alpha}^{J}\right\rangle$ is invertible and nonsingular. Based on this, we choose those $N_{P}$ exact solutions $\Psi_{\alpha}^{J}$ having the largest overlap with the two-particle model space states $\Phi_{a, b}^{J}$. With the solution $\omega$, the non-Hermitian effective interaction $R$ is given by $[26,27]$

$$
R=P \tilde{H} P-P H_{0} P=P V_{12} P+P V_{12} Q \omega .
$$

It would be preferable to obtain a complex symmetric effective interaction in order to take advantage of the antisymmetrization of the two-particle basis. This may be accomplished by the complex orthogonal transformation

$$
V_{\mathrm{eff}}=U^{-1}\left(H_{0}+V_{12}\right) U-H_{0},
$$

where $U$ is complex orthogonal and defined by

$$
U=\exp (-S), \quad S=\operatorname{arctanh}\left(\omega-\omega^{T}\right),
$$

and

$$
U^{T} U=U U^{T}=1, \quad U^{T}=U^{-1} .
$$

Such complex orthogonal transformations preserve the Berggren metric $x^{T} x$ of any vector $x \in\left\{C^{n}\right\}$. This feature allows us to define a complex symmetric effective two-body interaction

$$
V_{\mathrm{eff}}=\left(P+\omega^{\mathrm{T}} \omega\right)^{1 / 2}(P H P+P H Q \omega)\left(P+\omega^{\mathrm{T}} \omega\right)^{-1 / 2}-H_{0} .
$$

In the limit $N_{P}=N$, where $N$ is a dimension of the fully two-body problem, the effective interaction equals the "bare" interaction $V_{12}$. To determine $V_{\text {eff }}$ numerically, one has to find the square root of the matrix $A=\left(P+\omega^{\mathrm{T}} \omega\right)$. In the case of $A$ being real and positive definite, the method based on eigenvector decomposition gives generally a stable solution. For a complex matrix $A$, however, that method is generally numerically unstable. An approach suitable for complex matrices is based on properties of the matrix sign function. It can be shown that the square root of the matrix is related to the matrix sign function [38]. In the case of $A$ being complex and having all eigenvalues in the open right half complex plane, iterations based on the matrix sign function are generally more stable. Here we applied a stable iteration scheme for the matrix sign derived by Denman and Beavers [39].

Now we have at hand all the tools necessary for the numerical determination of an effective two-body interaction for use in Gamow shell-model calculations of ${ }^{7} \mathrm{He}$. Summarizing, the recipe is as follows:

- We first choose a set of single-particle orbits and divide them into a single-particle model space $p$ and a single-particle excluded space $q$.

- The single-particle orbits define, in turn, a two-body model space $P$ and a two-body excluded space $Q$.

- We diagonalize thereafter the two-body Schrödinger equation exactly and derive a model space effective interaction $V_{\text {eff }}$ by a similarity transformation.

- This two-body effective interaction $V_{\text {eff }}$ can, in turn, be used in a many-body context such as large-scale shell-model diagonalizations.
Below we apply the Lee-Suzuki similarity transformation method to the Gamow shell-model calculation of the ground state of ${ }^{7} \mathrm{He}$, for the case of $p_{3 / 2}$ single-particle motion only, fully diagonalized in Sec. II D. The three-particle model space is defined by all single-particle orbits belonging to $p$ in the same way as the two-particle model space was defined in Eq. (26). With the similarity transformed effective two-body interaction we can in turn solve the three-body eigenvalue problem, which reads

$$
\left(H_{0}+\sum_{i<j}^{3} V_{\mathrm{eff}}(i, j)\right)\left|\Psi_{\alpha}^{J}\right\rangle=E_{\alpha}\left|\Psi_{\alpha}^{J}\right\rangle
$$

Figures 8 and 9 show the convergence of the real and imaginary part of the $J^{\pi}=3 / 2^{-}$resonance in ${ }^{7} \mathrm{He}$, as the model space is increased. For comparison, we plot the results for a diagonalization within the model space using the "bare" interaction. Results from the effective interaction constructed with the similarity transformation method converge much faster than results obtained with the bare interaction. A satisfactory convergence is obtained with 10-11 single-particle Berggren states in the single-particle model space $p$ from ${ }^{5} \mathrm{He}$, corresponding to $\approx 700-800$ three-particle states $N_{P}$. Considering the full dimension of the three-particle problem is 9224 , we have drastically reduced the dimension to about $8 \%$ of the full space. This is a considerable benefit, which may allow us to extend the Gamow shell model with a complex scaled single-particle basis to heavier systems and realistic effective interactions. However, we can further improve this approach by considering perturbative techniques as well. Perturbative

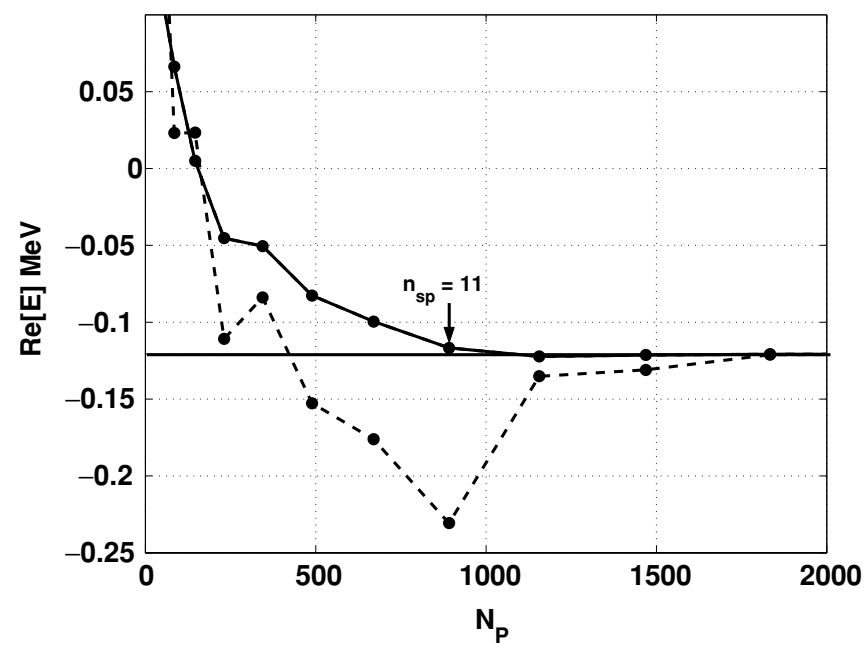

FIG. 8. Convergence of the real part of the $J^{\pi}=3 / 2^{-}$resonance in ${ }^{7} \mathrm{He}$ for a space defined by occupation of $p_{3 / 2}$ single-particle orbits only. The abscissa represents the number of three-particle model space configurations $N_{P}$, while $n_{\mathrm{sp}}$ represents the total number of single-particle momenta for the $p_{3 / 2}$ single-particle quantum numbers $l j$. The solid line corresponds to the effective interaction generated by the Lee-Suzuki similarity transformation method; the dashed line is obtained using the the bare interaction and the same number of three-body configurations. The $3 / 2^{-}$resonance is located at $E=$ $-(0.120731+0.122211 i) \mathrm{MeV}$. The horizontal line is the real energy obtained in the full space of three-body configurations. 


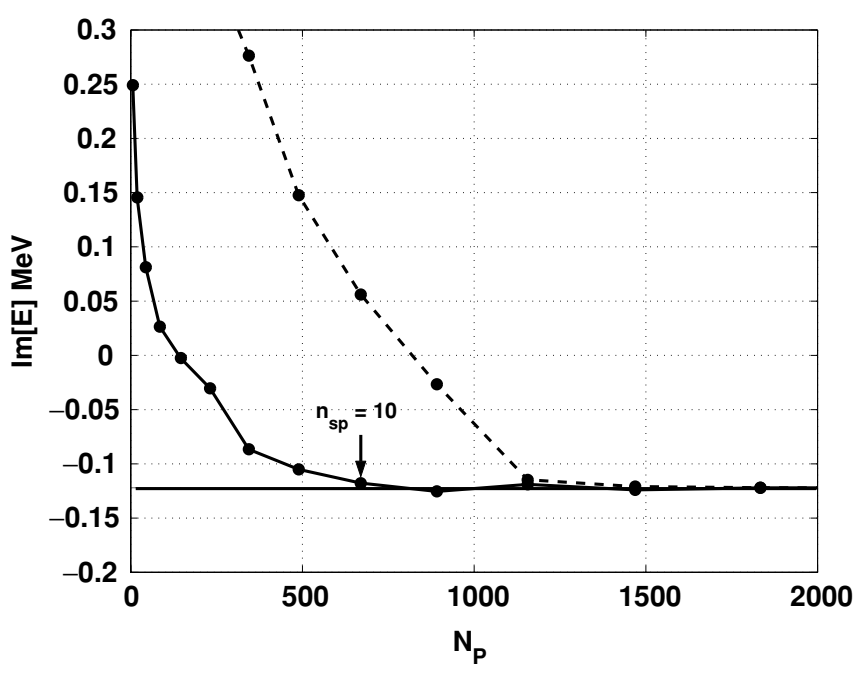

FIG. 9. Same as Fig. 8, but for the imaginary part.

techniques have been widely used in atomic, molecular, and nuclear many-body problems. They represent one of many possible approaches for reducing the dimensionality of the many-body problem. Below, we focus on recent approaches from quantum chemistry [30-32], where the emphasis is on scattering theory and electron decays in many-body systems.

In quantum chemistry, the interaction is rather well established and perturbative methods are viable possibilities. In the nuclear many-body problem, however, we need to renormalize the short-range part of the nucleon-nucleon and/or three-body force in order to obtain an effective interaction that can be used in, for example, shell-model studies. The Lee-Suzuki scheme has been successfully used for renormalizing the nucleonnucleon force and its short-range behavior. Other techniques such as the two-body $G$ matrix discussed in Ref. [40] serve much of the same purposes and can be used as effective interactions for nuclear many-body approaches. Such effective interactions, defined for large two-body spaces, are in turn used to generate medium dependent correlations using techniques such as the coupled cluster methods $[40,41]$ or many-body perturbation theory.

Our ultimate goal is to marry the Lee-Suzuki approach with either perturbative many-body techniques or coupled cluster methods in order to derive many-body correlations not present at the two-body level of Eq. (25). However, since much progress has been achieved in quantum chemistry for decaying systems based on perturbative many-body methods, we focus only on these techniques in the next section. In Sec. V we bring together the Lee-Suzuki scheme with the many-body perturbation theory discussed in Sec. IV and show that the combination of the two offers a viable approach to studying weakly bound systems.

\section{MULTIREFERENCE PERTURBATION METHOD}

The Möller-Plesset multireference perturbation theory method (MRPTM) has recently been revived in quantum chemistry [30-32], with emphasis on scattering theory and electron decays in many-body systems. Here we only give a brief outline of the method and refer the reader to Refs. [30-32] for further details. The basic idea of the multireference perturbation method is to first diagonalize within a small space (reference space) and then add a perturbation to the reference states by taking into account excitations from the reference space to the complement space. In the application of MRPTM to the formation of three-particle resonances in ${ }^{7} \mathrm{He}$, we first have to define a suitable three-particle reference (model) space. The chosen model space $P$ should ideally contain most of the correlations in the fully correlated three-particle wave function, hence the coupling of $P$ with the complement space $Q$ should be weak. Based on our knowledge from the two-particle system ${ }^{6} \mathrm{He}$, studied in Sec. II C, a reasonably good choice for the complement space $Q$ would consist of three-body configurations where all particles move in complex-continuum orbits. This is corroborated by studying the squared amplitudes of the three-particle configurations given in Table XIII for the ${ }^{7} \mathrm{He}$ ground state, where it is seen that the amplitudes of configurations in which all particles move in continuum orbits are small. In Refs. [6] and [7], where the helium isotopes ${ }^{6-9} \mathrm{He}$ were studied within the Gamow shell-model formulation, the authors reached similar conclusions for ${ }^{6} \mathrm{He}$ and ${ }^{7} \mathrm{He}$. The three-particle model space, and corresponding complement space, used in our MRPTM calculations of ${ }^{7} \mathrm{He}$ is then defined by

$$
P \equiv\left\{\begin{array}{c}
|R R R\rangle,|R R C\rangle,|R C C\rangle, \\
\operatorname{Re}\left(e_{a}+e_{b}+e_{c}\right)<E_{\mathrm{cut}}, \\
\operatorname{Im}\left(e_{a}+e_{b}+e_{c}\right)>-E_{\mathrm{cut}}
\end{array}\right\} Q=1-P
$$

where the $P$ space is given by configurations where at most two particles move in continuum orbits. In addition, $P$ is further defined by a rectangular cutoff in the complex energy plane. This cutoff in energy is motivated by our assumption that three-particle configurations high in energy play a minor role in the formation of low-lying resonances. Note that this way of defining a three-particle model space is different from the one used in the previous section, where the two- and three-particle model spaces were dictated by the single-particle model and complement space $p$ and $q$, respectively. In our MRPTM calculations of ${ }^{7} \mathrm{He}$, we start with the complete single-particle space $p+q$ and construct all $N=N_{P}+N_{Q}$ possible three-particle configurations for given spin and parity. Having constructed a complete three-particle antisymmetric basis, we divide the three-particle space into a model space $P$ and a complement space $Q$ by some given selection criterion; in our case, it is the criterion given in Eq. (43). Figure 10 gives a plot of the $J^{\pi}=3 / 2^{-}$unperturbed (noninteracting) three-particle spectrum of ${ }^{7} \mathrm{He}$, where at most two particles move in complex-continuum orbits; three different cutoffs in energies and corresponding model spaces are shown. Note that only $p_{3 / 2}$ single-particle orbitals are taken into account.

Although the coupling with pure continuum configurations turns out to be weak in our study of ${ }^{7} \mathrm{He}$, this may not always be the case. If three-body forces were included, one would expect an enhancement in the amplitude strengths of the $|C C C\rangle$ configurations, since three-body matrix elements of the type 


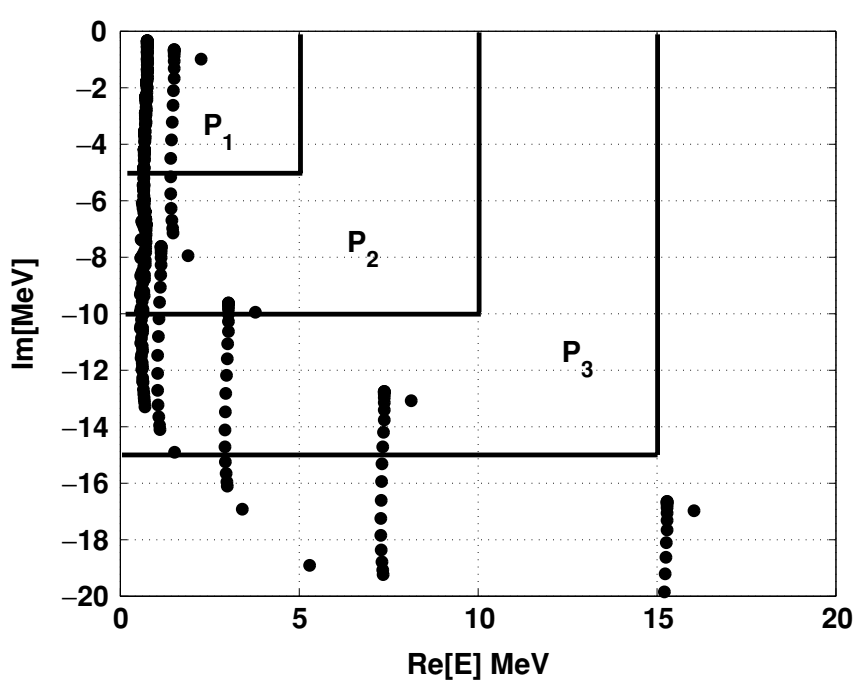

FIG. 10. Three choices of the model space used in the multiconfiguration perturbation method. The three-particle model space states are constructed such that at most two particles move in the nonresonant continuum.

$\langle R R R|V| C C C\rangle$ are no longer vanishing. Furthermore, even at the level of two-body interactions, the multiparticle resonances may have a considerable coupling with pure nonresonant continuum configurations. Investigators [9] have found that in the case of two-particle resonances in ${ }^{11} \mathrm{Li}$, the continuum contributions play in certain cases a more important role than the single-particle resonance orbitals. In principle, this does not pose a significant problem regarding the applicability of MRPTM to Gamow shell-model calculations. The three-particle model space originally defined by Eq. (43) may be redefined to include any number of pure nonresonant continuum configurations $|C C C\rangle$, say, for example, the $N_{C}$ configurations $|C C C\rangle$ closest to the three-particle breakup threshold $(E=0 \mathrm{MeV})$.

Having defined a suitable three-body model space, the shell-model eigenvalue problem takes the following form

$$
\left(\begin{array}{cc}
H^{P P} & H^{P Q} \\
H^{Q P} & H^{Q Q}
\end{array}\right)\left(\begin{array}{c}
P \Psi_{\alpha}^{J} \\
Q \Psi_{\alpha}^{J}
\end{array}\right)=E_{\alpha}^{J}\left(\begin{array}{c}
P \Psi_{\alpha}^{J} \\
Q \Psi_{\alpha}^{J}
\end{array}\right),
$$

where the matrix elements of the model space block are given by

$$
H_{i, j}^{P P}=\left\langle\tilde{\Phi}_{i}^{J}|H| \Phi_{j}^{J}\right\rangle=\left\langle\tilde{\Phi}_{(a b) c}^{J}|H| \Phi_{(d e) f}^{J}\right\rangle,
$$

with $\tilde{\Phi}_{(a b) c}^{J} \in P$ and $\Phi_{(d e) f}^{J} \in P$. The matrix elements of the remaining blocks are given in the same form, with $\Phi_{(a b) c}^{J}$ belonging either to $P$ or $Q$. See the Appendix for details on the evaluation of three-body matrix elements with two-body forces in $j-j$ coupling. Thereafter, the full Hamiltonian is divided into two parts:

$$
\begin{aligned}
\left(\begin{array}{ll}
H^{P P} & H^{P Q} \\
H^{Q P} & H^{Q Q}
\end{array}\right) & =\left(\begin{array}{cc}
H^{P P} & 0 \\
0 & D^{Q Q}
\end{array}\right)+\left(\begin{array}{cc}
0 & H^{P Q} \\
H^{Q P} & \tilde{H}^{Q Q}
\end{array}\right) \\
& =H^{0}+H^{1} .
\end{aligned}
$$

Here $D^{Q Q}$ is the diagonal part and $\tilde{H}^{Q Q}$ the off-diagonal part of $H^{Q Q}$. Writing it in this form shows that $H^{0}$ defines the unperturbed part while $H^{1}$ gives the perturbations to $H^{0}$. Provided $H^{0}$ is nonsingular, the model space block $H^{P P}$ may be decoupled by constructing a complex orthogonal matrix $\mathbf{C}$ which diagonalizes $H^{0}$, i.e., $\mathbf{C} H^{0} \mathbf{C}^{T}=$ $\operatorname{Diag}\left(E_{1}^{0}, E_{2}^{0}, \ldots, E_{N}^{0}\right)$. Since $H^{0}$ is a block diagonal matrix, the matrix $\mathbf{C}$ is given in the form

$$
\mathbf{C}=\left[\begin{array}{ll}
\chi & 0 \\
\mathbf{0} & 1
\end{array}\right] \text {. }
$$

A more convenient three-particle basis that decouples the reference space is then given by

$$
\Upsilon_{i}^{J}=\sum_{j=1}^{N} C_{i, j} \Phi_{j}^{J}=\left\{\begin{array}{c}
\sum_{j=1}^{N_{P}} \chi_{i, j} \Phi_{j}^{J}, \quad i=1, N_{P}, \\
\Phi_{i}^{J}, \quad i=N_{P}+1, N .
\end{array}\right.
$$

The complex orthogonal matrix $\chi$ which spans the reference space $P$ defines our new set of reference states.

Identifying the reference state $\Upsilon_{i}^{J}, \quad i \in\left(1, \ldots, N_{P}\right)$, which gives the zeroth-order approximation of the exact threeparticle resonance, may be done by determining which state $\Upsilon_{i}^{J}$ has the largest overlap with the pure pole configuration $\left|\Phi_{\text {res }}^{J}\right\rangle=|R R R\rangle$,

$$
\operatorname{Max}\left\{\left|\left\langle\tilde{\Phi}_{\text {res }}^{J} \mid \Upsilon_{i}^{J}\right\rangle\right|\right\}_{i=1}^{N_{P}}=\operatorname{Max}\left\{\left|\chi_{i, j=\text { res }}\right|\right\}_{i=1}^{N_{P}} .
$$

In our MRPTM calculation of ${ }^{7} \mathrm{He}$, we used the algorithm defined in Eq. (49) as the identification method. To make sure that we picked out the "correct" physical state, we studied the complex energy trajectories as the interaction was gradually turned on. As a measure of how well the zeroth-order three-particle resonance wave function $\Upsilon_{i=\text { res }}^{J}$ resembles the exact resonance wave function, one can calculate the complex variance $\sigma_{c}^{2}[24,42]$,

$$
\begin{aligned}
\sigma_{c}^{2}(i=\mathrm{res}) & =\left\langle\Upsilon_{i}^{J *}\left|\left(H-E_{i}^{0}\right)^{2}\right| \Upsilon_{i}^{J}\right\rangle \\
& =\chi_{i}^{T} H^{P Q} H^{Q P} \chi_{i},
\end{aligned}
$$

where $\chi_{i}$ labels the $i$ th column of the $N_{P} \times N_{P}$ matrix $\chi$. The reference states $\Upsilon_{i}^{J}$ are normalized. In Ref. [42], it was proved that the complex variance $\sigma_{c}$ provides an upper bound to the exact resonance energy,

$$
\left|E_{\text {res }}^{\text {exact }}-E_{\text {res }}^{0}\right| \leqslant\left|\sigma_{c}\right| \text {. }
$$

The upper bound provides us with a valuable tool for determining how close the approximated resonance energy is to the exact resonance energy, especially since in most practical applications the exact resonance energy is not known. However, in this particular case, we know the exact position of the resonance energy from the full diagonalization in Sec. II D, so we can compute $\left|E_{\text {res }}^{\text {exact }}-E_{\text {res }}^{0}\right|$ and compare with $\left|\sigma_{c}\right|$. Table XIV gives the zeroth-order resonance energy and corresponding upper bounds $\left|\sigma_{c}\right|$ of the $J^{\pi}=3 / 2^{-}$ground state of ${ }^{7} \mathrm{He}$, using the three-particle model space defined in Eq. (43) for several different energy cutoffs.

As Table XIV indicates, a diagonalization within a model space where at most two particles move in continuum orbits does not provide a satisfactory approximation of the 
TABLE XIV. Convergence of the zeroth-order energy of the $J^{\pi}=$ $3 / 2^{-}$ground state of ${ }^{7} \mathrm{He}$ for increasing energy cutoffs within the reference space; see Eq. (43). The corresponding upper bounds $\left|\sigma_{c}\right|$ of the exact ground state energy are also given. The numbers $N_{P}$ and $N_{Q}$ give the dimensions of the three-particle model space $P$ and complement space $Q$, respectively. The full dimensionality with $n_{\mathrm{sp}}=24$ is $N=N_{P}+N_{Q}=9224$.

\begin{tabular}{rrrrccc}
\hline \hline$E_{\text {cut }}$ & $N_{P}$ & $N_{Q}$ & $\operatorname{Re}\left[E_{\text {res }}^{0}\right]$ & $\operatorname{Im}\left[E_{\text {res }}^{0}\right]$ & $\left|E_{\text {res }}^{\text {exact }}-E_{\text {res }}^{0}\right|$ & $\left|\sigma_{c}\right|$ \\
\hline 0 & 1 & 9223 & 0.970 & 1.505 & 1.959 & 2.722 \\
10 & 305 & 8919 & -0.292 & -0.045 & 0.188 & 1.891 \\
20 & 403 & 8821 & -0.198 & -0.183 & 0.098 & 1.479 \\
30 & 469 & 8755 & -0.145 & -0.153 & 0.039 & 0.841 \\
50 & 479 & 8745 & -0.136 & -0.157 & 0.038 & 0.618 \\
100 & 543 & 8681 & -0.135 & -0.157 & 0.038 & 0.600 \\
1000 & 1083 & 8141 & -0.135 & -0.157 & 0.038 & 0.600 \\
\hline \hline
\end{tabular}

exact ground state energy of ${ }^{7} \mathrm{He}$ within our model. Having obtained the zeroth-order approximation to the exact threeparticle resonance, after full diagonalization of the unperturbed Hamiltonian $H^{0}$, we may perform a standard perturbation expansion in energy to take into account couplings with configurations where all particles move in continuum orbits. Using intermediate normalization, the energy corrections up to third order for a given state $\Upsilon_{i}^{J}, \quad i \in\left(1, \ldots, N_{P}\right)$ may be shown to be [30-32],

$$
\begin{aligned}
E_{i}^{0} & =\chi_{i}^{T} H^{P P} \chi_{i}, \quad E_{i}^{1}=0, \\
E_{i}^{2} & =\sum_{j=1}^{N_{Q}} \frac{M_{i, j}^{2}}{E_{i}^{0}-D_{j, j}^{Q Q}}, \\
E_{i}^{3} & =\sum_{j, k=1}^{N_{Q}} \frac{M_{i, j} \tilde{H}_{j, k}^{Q Q} M_{i, k}}{\left(E_{i}^{0}-D_{j, j}^{Q Q}\right)\left(E_{i}^{0}-D_{k, k}^{Q Q}\right)}, \quad j \neq k,
\end{aligned}
$$

where we have defined the $N_{P} \times N_{Q}$ matrix $\mathbf{M}=\chi^{T} H^{P Q}$, and $\chi_{i}$ means the $i$ th column of the matrix $\chi$. For the perturbation series to converge, the reference energies $E_{i}^{0}$ must be separated from the diagonal elements $D_{k, k}^{Q Q}$. This can in principle always be enforced by enlarging the model space $P$, for example, by including a set of pure complex-continuum configurations in $P$.

Observe that there is no first-order correction to the energy, meaning that it has been accounted for by the reference states and energies $\Upsilon_{i}^{J}, E_{i}^{0}$. Note also that we used the bare two-body interaction of Eq. (16). This is to be contrasted with the method outlined in the next section, where we use the Lee-Suzuki transformation to define an effective interaction.

Figures 11 and 12 show the convergence of the real and imaginary part of the three-particle resonance energy in the multi-reference perturbation method up to third order; see Eqs. (52), (53), and (54). The model space used here is given in Eq. (43), and the calculations were done for increasing energy cutoffs $E_{\text {cut }}=0,5, \ldots, 30 \mathrm{MeV}$. Here only $p_{3 / 2}$ single-particle orbitals were considered, and the calculations used 24 integration points in the construction of the single-

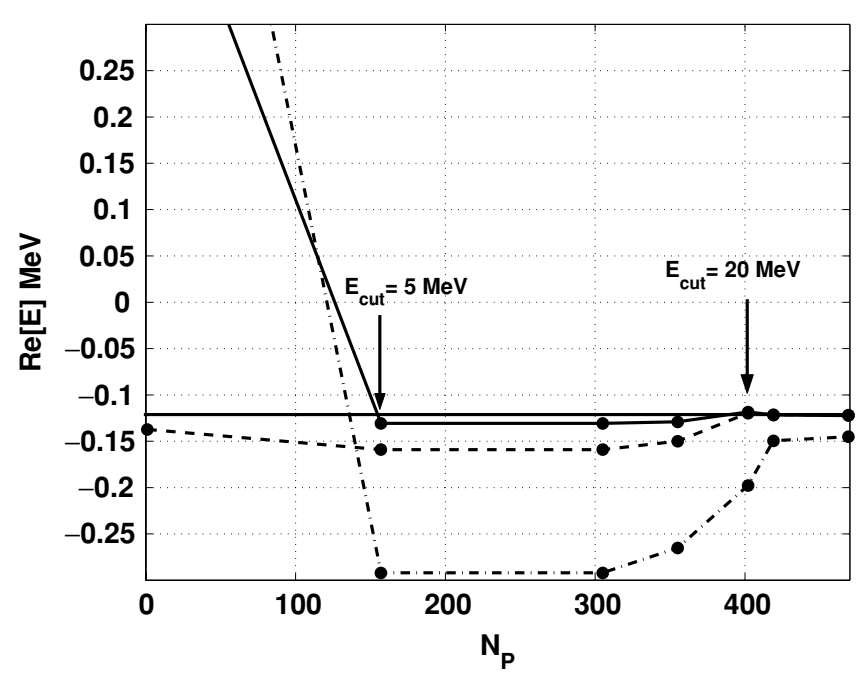

FIG. 11. Convergence of the real part of the $J^{\pi}=3 / 2^{-}$resonance in ${ }^{7} \mathrm{He}$, as the dimension of three-particle model space increases with increasing cutoff in energy. The energy cutoff is increased in steps of $5 \mathrm{MeV}$, i.e., $E_{\mathrm{cut}}=0,5, \ldots, 30$, and given by the filled circles. The horizontal line indicates the real part of the $J^{\pi}=3 / 2^{-}$resonance located at $E=-(0.120731+0.122211 i) \mathrm{MeV}$. The dashed-dotted line is the zeroth-order energy, the dashed line represents the secondorder energy, and the solid line is the third-order energy.

particle basis for ${ }^{5} \mathrm{He}$, giving in total $N=N_{P}+N_{Q}=9224$ three-particle configurations for ${ }^{7} \mathrm{He}$, with spin and parity $J^{\pi}=3 / 2^{-}$. The convergence of the ground state energy is plotted with respect to the number of three-particle model space states $N_{P}$ for each energy cutoff. From Figs. 11 and 12, one notes that a satisfactory convergence is obtained with $N_{P} \approx 400$, corresponding to the energy cutoff $E_{\text {cut }}=20 \mathrm{MeV}$. Excitations of model space configurations located above $E_{\text {cut }} \approx 5 \mathrm{MeV}$ yield small contributions to the second- and third-order corrections to the resonance energy, as expected. Observe that the second- and third-order terms converge at

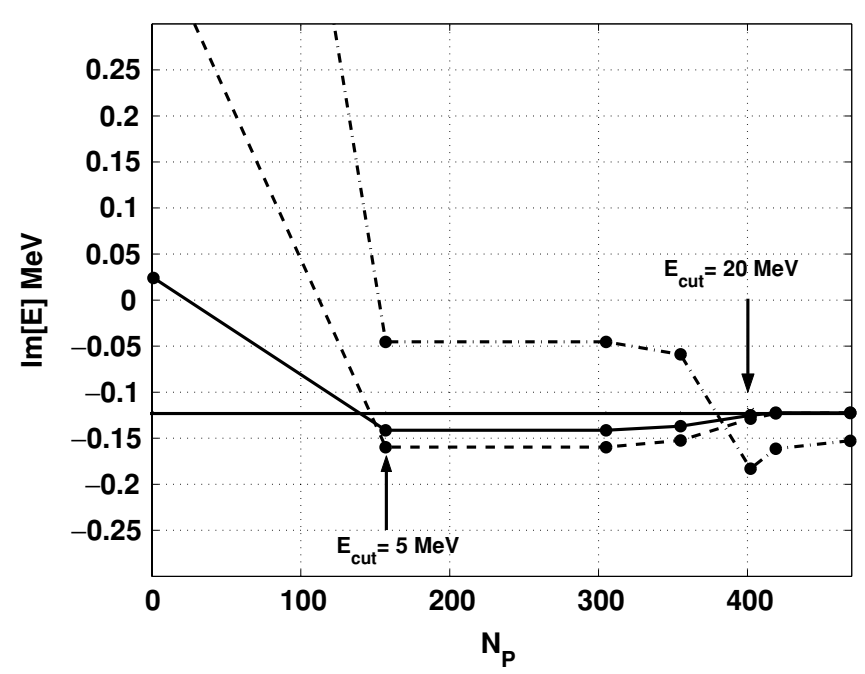

FIG. 12. Same as Fig. 11, but for the imaginary part of the $J^{\pi}=$ $3 / 2^{-}$resonance in ${ }^{7} \mathrm{He}$. 
the same number of model space states, which indicates that second-order corrections in energy are apparently sufficient for our applications. This is also an advantage from a numerical point of view. In second order, one has to store only the diagonal part of the block $H^{Q Q}$, while in third order the complete block $H^{Q Q}$ has to be stored, which may be extremely large in many cases. The zeroth-order energy, which corresponds to diagonalization within $P$, does not saturate at the exact resonance energy with increasing $N_{P}$, which again shows that possible couplings with the $Q$ space have to be accounted for to obtain accurate calculations.

Summing up these results, we see that we obtain stable results with approximately $N_{P} \approx 400$ three-body configurations within the multireference perturbation method, while the similarity transformation method of Sec. III gives stable results for $N_{P} \approx 800$ three-body configurations for the same problem. The question now is whether we can marry these two approaches in our quest for smaller Gamow shell-model spaces. Such a combined approach also forms the standard approach in nuclear shell-model studies, either based on no-core approaches or with effective interactions for valence systems.

This is the topic of the next section.

\section{EFFECTIVE INTERACTION SCHEME FOR THE GAMOW SHELL MODEL}

The previous sections demonstrated that the Lee-Suzuki similarity transformation and the multireference perturbation method may both be used in the Gamow shell model to account for the most important correlations of a multiparticle resonance. Although the dimensionality of the problem derived from these methods was significantly reduced compared to that of the full problem, large dimensionalities may still be a severe problem when dealing with more than three particles in a big valence space.

The drawback of the multireference perturbation method is that one has to store extremely large matrices $H^{Q Q}$ if one wishes to go beyond second order in perturbation theory. In the similarity transformation method, one does not have to deal with $H^{Q Q}$, as couplings with the $Q$-space states have been dealt with in practical calculations at least at the two-body level. Going to systems with larger degrees of freedom, the $P$ space may, nevertheless, at the converged level, be too large for our brute force diagonalization approach.

In this section, we propose an effective interaction and perturbation theory scheme for the Gamow shell model. This approach combines the similarity transformation method and the multireference perturbation method, so that multiparticle resonances-where several particles move in large valence spaces - may be calculated without a diagonalization in the full space. Our ultimate goal is to derive effective interactions for weakly bound systems to be used with the coupled cluster methods [40,41].

Our algorithm is as follows:

1. Choose an optimal set of $n_{\mathrm{sp}}$ single-particle orbits, which in turn defines two-body $P_{2 p}$ and many-body spaces. In our test case these single-particle orbits are defined by selected states in ${ }^{5} \mathrm{He}$.

2. Construct a two-particle effective interaction by the LeeSuzuki similarity transformation method within the twoparticle model space $P_{2 p}$. Such diagonalizations can be done for very large spaces [34-37].

3. The next step is to divide the multiparticle model space $P$ in two smaller spaces $P^{\prime}$ and $Q^{\prime}$, where $P=P^{\prime}+Q^{\prime}$ and $N_{P}=N_{P^{\prime}}+N_{Q^{\prime}}$. The choice of $P^{\prime}$ should be dictated by our knowledge of the physical system. As an example, one may consider those single-particle configurations within the $P$ space that play the dominant role in the formation of the multiparticle resonance. The number $N_{P}=N_{P^{\prime}}+N_{Q^{\prime}}$ represents the total number of many-body configurations within the $P$ space.

4. Now that we have divided the $P$ space into two subspaces $P^{\prime}$ and $Q^{\prime}$, we use, for example, the multireference perturbation method to account for excitations from the $P^{\prime}$ space to the $Q^{\prime}$ space to obtain energy corrections to a specific order. We increase the size of the $P^{\prime}$ space until convergence is obtained. In the case $N_{P^{\prime}}=N_{P}$ and $N_{Q^{\prime}}=N_{P}-N_{P^{\prime}}=0$, the multireference perturbation expansion terminates at zeroth order and corresponds to a full diagonalization within the $P$ space. Another option is to use, for example, the coupled cluster method as exposed in Refs. [40,41].

5. Start from the top again with a larger set of singleparticle orbits and continue until a convergence criterion is reached.

We illustrate these various choices of model spaces in the following two figures. Figure 13 defines our model space for the Lee-Suzuki similarity transformation at the two-body level. This corresponds to steps one and two in the above algorithm. The set of single-particle orbits defines the last single-particle orbit in the model space $n_{\mathrm{sp}}$. Note that we could have chosen a model space defined by a cut in energy, as done by the No-Core Collaboration [34-37]. Figure 14 demonstrates a possible division of the three- and many-particle space

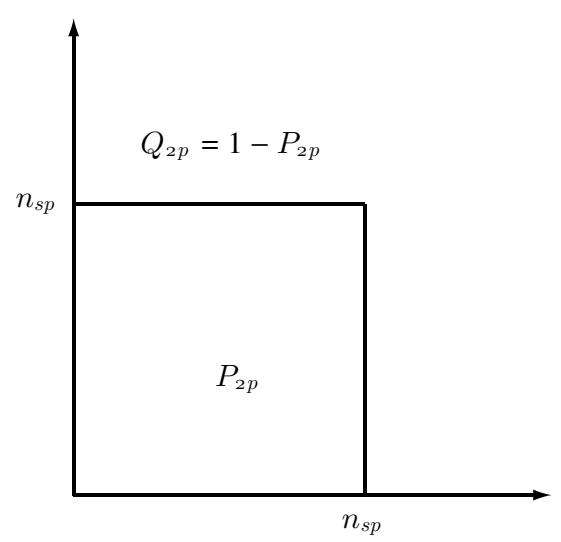

FIG. 13. Possible definition of the two-body exclusion operator $Q_{2 p}=1-P_{2 p}$ used to compute the Lee-Suzuki similarity transformation and its effective interaction at the two-body level. The border of the model space is defined by the last single-particle orbit $n_{\text {sp. }}$. 


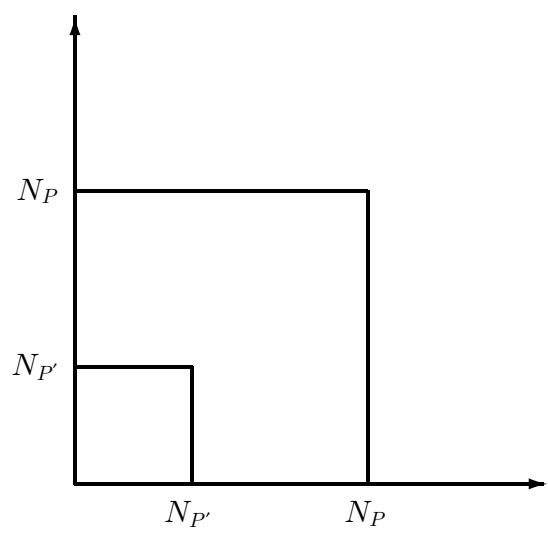

FIG. 14. Possible definition of many-body space $N_{P}$ and reduced space $N_{P}^{\prime}$.

into the full model space $P$ and a smaller space $P^{\prime}$. These figures serve only to illustrate the method. In our actual calculations, we define the smaller space $P^{\prime}$ via an energy cut in the real and imaginary eigenvalues and selected many-body configurations.

In summary, defining a set of single-particle orbits in order to construct the two- and many-body model spaces, we obtain first an effective two-body interaction in the space $P_{2 p}$ by performing the Lee-Suzuki [26-29] transformation. This interaction and the pertinent single-particle orbits are then used to define a large many-body space. It is therefore of interest to see if we can reduce this dimensionality through the definition of smaller spaces and perturbative corrections.

We present here as a test case the calculation of the $J^{\pi}=3 / 2^{-}$three-particle resonance within the perturbation scheme outlined above. Twenty-four single-particle orbits for the $l j$ configuration $p_{3 / 2}$ are included, giving a total dimension of $d=9224$ for the $J=3 / 2$ three-particle basis. We define five different three-particle model spaces $P$, given by the total number of three-body configurations $N_{P}$. The number of single-particle orbits and three-body states are listed in Table XV. The single-particle model space, defining $P$, is constructed according to the prescription outlined in Sec. III.

For each three-particle model space $P$ listed in Table $\mathrm{XV}$, a division into two smaller subspaces $P^{\prime}$ and $Q^{\prime}$ is performed, i.e., $P=P^{\prime}+Q^{\prime}$. The subspace $P^{\prime}$ that defines a proper

TABLE XV. Five different $P$ spaces defined for increasing number of single-particle model space orbits $n_{\mathrm{sp}}$ consisting of the $l j$ configuration $p_{3 / 2}$. The number $N_{P}$ gives the dimension of the threeparticle model space $P$ for $J^{\pi}=3 / 2^{-}$with a full dimensionality with $n_{\text {sp }}=24$ of $N=9224$.

\begin{tabular}{rrrrrr}
\hline \hline$P$ & $P_{1}$ & $P_{2}$ & \multicolumn{1}{c}{$P_{3}$} & $P_{4}$ & $P_{5}$ \\
\hline$n_{\text {sp }}$ & 8 & 10 & 12 & 14 & 16 \\
$N_{P}$ & 344 & 670 & 1156 & 1834 & 2736 \\
\hline \hline
\end{tabular}

TABLE XVI. Resonance energy to second $\left(E^{2}\right)$ and third $\left(E^{3}\right)$ order in the multireference perturbation expansion, for model space $P_{1}$ given in Table $\mathrm{XV}$. The subspaces $P^{\prime}{ }_{1}$ are defined for different energy cutoffs, increased in steps of $10 \mathrm{MeV}$. The last line gives the exact energy within $P_{1}$. Energies are in $\mathrm{MeV}$.

\begin{tabular}{rrrrrrr}
\hline \hline \multicolumn{3}{c}{$N_{P_{1}}=344$} & \multicolumn{3}{c}{$N_{P_{1 \max }^{\prime}}=113$} \\
\hline$N_{P^{\prime}{ }_{1}}$ & $N_{Q_{1}^{\prime}}$ & $E_{\text {cut }}$ & $\operatorname{Re}\left[E^{2}\right]$ & $\operatorname{Im}\left[E^{2}\right]$ & $\operatorname{Re}\left[E^{3}\right]$ & $\operatorname{Im}\left[E^{3}\right]$ \\
\hline 1 & 343 & 0 & 0.066 & 0.322 & 0.606 & 0.088 \\
113 & 231 & 10 & 0.041 & -0.075 & 0.041 & -0.076 \\
\hline \multicolumn{1}{l}{ Exact within $P_{1}:$} & & 0.042 & -0.076 & & \\
\hline \hline
\end{tabular}

subset of each model space $P$ is defined by

$$
P^{\prime}{ }_{i} \equiv\left\{\begin{array}{c}
|R R R\rangle,|R R C\rangle,|R C C\rangle \\
\operatorname{Re}\left(e_{a}+e_{b}+e_{c}\right)<E_{\mathrm{cut}} \\
\operatorname{Im}\left(e_{a}+e_{b}+e_{c}\right)>-E_{\mathrm{cut}}
\end{array}\right\} \subset P_{i} .
$$

Having defined the spaces $P, Q, P^{\prime}$, and $Q^{\prime}$, we write the Hamiltonian within the $P$ space in the form

$$
\begin{aligned}
\left(\begin{array}{ll}
H^{P^{\prime} P^{\prime}} & H^{P^{\prime} Q^{\prime}} \\
H^{Q^{\prime} P^{\prime}} & H^{Q^{\prime} Q^{\prime}}
\end{array}\right) & =\left(\begin{array}{cc}
H^{P^{\prime} P^{\prime}} & 0 \\
0 & D^{Q^{\prime} Q^{\prime}}
\end{array}\right)+\left(\begin{array}{cc}
0 & H^{P^{\prime} Q^{\prime}} \\
H^{Q^{\prime} P^{\prime}} & \tilde{H}^{Q^{\prime} Q^{\prime}}
\end{array}\right) \\
& =H^{0}+H^{1} .
\end{aligned}
$$

Where $H^{P^{\prime} P^{\prime}}=P^{\prime} H^{P P} P^{\prime}, H^{P^{\prime} Q^{\prime}}=P^{\prime} H^{P P} Q^{\prime}, H^{Q^{\prime} P^{\prime}}=$ $Q^{\prime} H^{P P} P^{\prime}$, and $H^{Q^{\prime} Q^{\prime}}=Q^{\prime} H^{P P} Q^{\prime}$. Then we construct a matrix $C$ which diagonalizes $H^{0}$ and which defines our reference states within $P^{\prime}$; see Sec. IV for further details. Corrections to the reference states coming from correlations contained in $H^{1}$ are then added perturbatively up to third order in our case; see Eqs. (52)-(54). Tables XVI-XX give the ground state energy of ${ }^{7} \mathrm{He}$ up to second and third order in our MRPTM calculations within each model space $P_{i}$ given in Table XV. Note that for each space $P_{1}, P_{2}$, and so forth, listed in Table $\mathrm{XV}$, we compare the results from this perturbative analysis with those from the exact diagonalization done in these spaces.

As the number of reference states $N_{P^{\prime}}$ increases with increasing $E_{\text {cut }}$, one reaches a maximum of reference states $N_{P_{\max }^{\prime}}$ within each $P$ space. From the definition of the reference space $P^{\prime}$ in Eq. (55), it will never coincide with the $P$ space as one exhausts the number of configurations $|R R R\rangle,|R R C\rangle,|R C C\rangle$ within $P$, since by definition one never includes the configurations $|C C C\rangle$, i.e., $\left\{\left|P^{\prime}\right\rangle\right\} \subset\{|P\rangle\}$.

TABLE XVII. Same as Table XVI, but for model space $P_{2}$ and subspaces $P_{2}^{\prime}$.

\begin{tabular}{rrrrrrr}
\hline \hline & \multicolumn{3}{c}{$N_{P_{2}}=670$} & \multicolumn{3}{c}{$N_{P_{2 \max }^{\prime}}=181$} \\
\hline$N_{P_{2}^{\prime}}$ & $N_{Q_{2}^{\prime}}$ & $E_{\text {cut }}$ & $\operatorname{Re}\left[E^{2}\right]$ & $\operatorname{Im}\left[E^{2}\right]$ & $\operatorname{Re}\left[E^{3}\right]$ & $\operatorname{Im}\left[E^{3}\right]$ \\
\hline 1 & 669 & 0 & -0.053 & 0.357 & 0.562 & 0.059 \\
157 & 513 & 10 & -0.078 & -0.110 & -0.079 & -0.110 \\
181 & 489 & 20 & -0.082 & -0.110 & -0.083 & -0.110 \\
\hline \multicolumn{2}{l}{ Exact within $P_{2}:$} & -0.081 & -0.110 & & \\
\hline \hline
\end{tabular}


TABLE XVIII. Same as Table XVI, but for model space $P_{3}$ and subspaces $P_{3}^{\prime}$.

\begin{tabular}{rrrrrrr}
\hline \hline & \multicolumn{3}{c}{$N_{P_{3}}=1156$} & \multicolumn{3}{c}{$N_{P_{3 \max }^{\prime}}=265$} \\
\hline$N_{P_{3}^{\prime}}$ & $N_{Q_{3}^{\prime}}$ & $E_{\text {cut }}$ & $\operatorname{Re}\left[E^{2}\right]$ & $\operatorname{Im}\left[E^{2}\right]$ & $\operatorname{Re}\left[E^{3}\right]$ & $\operatorname{Im}\left[E^{3}\right]$ \\
\hline 1 & 1155 & 0 & -0.099 & 0.378 & 0.561 & 0.050 \\
205 & 951 & 10 & -0.114 & -0.134 & -0.114 & -0.133 \\
265 & 891 & 20 & -0.117 & -0.130 & -0.118 & -0.130 \\
\hline \multicolumn{7}{l}{ Exact within $P_{3}:$} \\
\hline \hline
\end{tabular}

The perturbation scheme for a reference space $P^{\prime}$ given by Eq. (55) will therefore only yield convergent results as long as our assumption holds that the configurations $|C C C\rangle$ play a minor role compared to the reference states. Although the configurations $|C C C\rangle$ turn out to play a minor role for the states we have considered in this work, there is no a priori reason for this to be the case when considering other multiparticle resonances. If no convergence is observed, one should simply choose another reference space $P^{\prime}$, based, for example, on the single-particle model space (see Figs. 13 and 14).

To graphically illustrate the perturbation scheme outlined above, Figs. 15 and 16 exhibit the results obtained in Tables XVI-XX. Figure 15 plots the real part of the ground state energy of ${ }^{7} \mathrm{He}$ up to third order, (column six in the tables) as the number of reference states $N_{P_{i}^{\prime}}$ increases with increasing $E_{\text {cut }}$. Figure 16 gives corresponding results for the imaginary part of the energy (column seven of the tables). From the plots and the tables we conclude that convergence is obtained for a small number of reference states $N_{P^{\prime}}$ $\sim 350-400$.

In the approach considered above, the dimension of the $Q^{\prime}$ space is considerably smaller than the dimension of the complement space $Q=1-P$. This makes computing the matrix elements of $H^{Q^{\prime} Q^{\prime}}$ much less time and memory consuming. We have seen from the above calculations that a termination of the perturbation expansion at second order compares well with the rate of convergence for the thirdorder expansion. This makes it numerically feasible to treat systems where several particles move in a large valence space, within the perturbative scheme outlined above combined with an effective two-body interaction derived from the Lee-Suzuki scheme. As such, we have a recipe for weakly

TABLE XIX. Same as Table XVI, but for model space $P_{4}$ and subspaces $P_{4}^{\prime}$.

\begin{tabular}{rrrrrrr}
\hline \hline & \multicolumn{3}{c}{$N_{P_{4}}=1834$} & \multicolumn{3}{c}{$N_{P_{4 \max }^{\prime}}=365$} \\
\hline$N_{P_{4}^{\prime}}$ & $N_{Q_{4}^{\prime}}$ & $E_{\text {cut }}$ & $\operatorname{Re}\left[E^{2}\right]$ & $\operatorname{Im}\left[E^{2}\right]$ & $\operatorname{Re}\left[E^{3}\right]$ & $\operatorname{Im}\left[E^{3}\right]$ \\
\hline 1 & 1833 & 0 & -0.134 & 0.397 & 0.532 & 0.026 \\
253 & 1581 & 10 & -0.155 & -0.160 & -0.130 & -0.141 \\
347 & 1487 & 20 & -0.119 & -0.127 & -0.120 & -0.126 \\
365 & 1469 & 30 & -0.122 & -0.123 & -0.123 & -0.124 \\
\hline Exact within $P_{4}:$ & -0.121 & -0.124 & & \\
\hline \hline
\end{tabular}

TABLE XX. Same as Table XVI, but for model space $P_{5}$ and subspaces $P_{5}^{\prime}$.

\begin{tabular}{rrrrrrr}
\hline \hline \multicolumn{3}{c}{$N_{P_{5}}=2736$} & \multicolumn{3}{c}{$N_{P_{5_{\max }^{\prime}}}=419$} \\
\hline$N_{P_{5}^{\prime}}$ & $N_{Q_{5}^{\prime}}$ & $E_{\text {cut }}$ & $\operatorname{Re}\left[E^{2}\right]$ & $\operatorname{Im}\left[E^{2}\right]$ & $\operatorname{Re}\left[E^{3}\right]$ & $\operatorname{Im}\left[E^{3}\right]$ \\
\hline 1 & 2735 & 0 & -0.137 & 0.399 & 0.530 & 0.024 \\
253 & 2483 & 10 & -0.159 & -0.160 & -0.131 & -0.141 \\
347 & 2389 & 20 & -0.120 & -0.129 & -0.118 & -0.125 \\
409 & 2327 & 30 & -0.122 & -0.122 & -0.122 & -0.122 \\
419 & 2317 & 40 & -0.122 & -0.122 & -0.123 & -0.122 \\
\hline \multicolumn{7}{l}{ Exact within $P_{5}:$} \\
\hline \hline
\end{tabular}

bound systems which preserves much of the methodology used in deriving effective interactions for the nuclear shell model.

\section{APPLICATION TO ${ }^{7}$ He INCLUDING BOTH $p_{1 / 2}$ AND $p_{3 / 2}$}

As shown in the previous sections, the effective interaction and perturbation scheme drastically reduces the dimensionality of the shell-model equations. Concluding this work, we apply the perturbation scheme to the calculation of the threeparticle resonances in ${ }^{7} \mathrm{He}$, where 24 single-particle orbits for each of the $l j$ single-particle orbits $p_{1 / 2}$ and $p_{3 / 2}$ now are included. The Hamiltonian for the $J^{\pi}=1 / 2^{-}, 3 / 2^{-}$and $J^{\pi}=$ $5 / 2^{-}$states for ${ }^{7} \mathrm{He}$ implies dimensions $N_{P}=29648,38896$ and $N_{P}=27072$, respectively. The main components of the

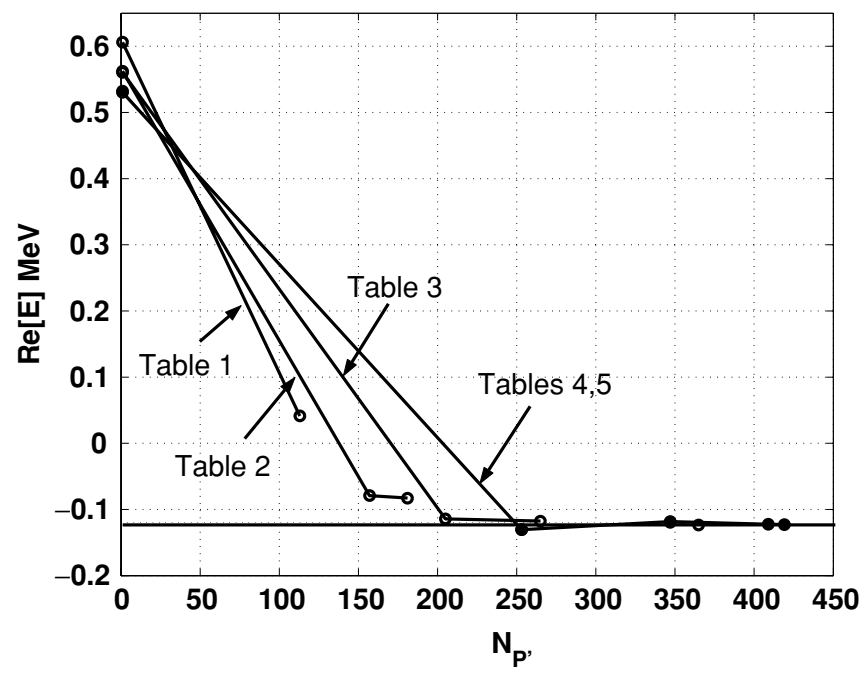

FIG. 15. Convergence of the real part of the $J^{\pi}=3 / 2^{-}$resonance energy in ${ }^{7} \mathrm{He}$ up to third order within the perturbative scheme outlined in the text, for the different model spaces $P$ given in Table XV. The open circles along the different solid lines give the calculations within each $P_{i} . N_{P^{\prime}}$ gives the number of reference states in $P^{\prime}$, which is a subspace of $P$. The horizontal line is the the real part of the $J^{\pi}=3 / 2^{-}$resonance located at $E=-(0.120731+$ $0.122211 i$ ) MeV. 


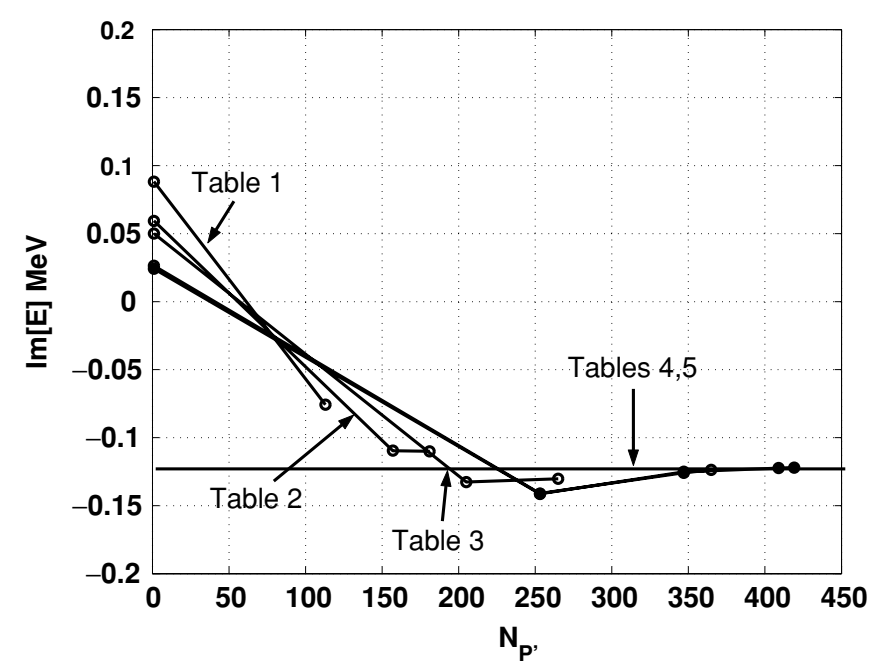

FIG. 16. Same as Fig. 15, but for the imaginary part.

${ }^{7} \mathrm{He}$ resonant wave functions turn out to be the of $|R R R\rangle$ type, in the noninteracting limit corresponding to energies and wave functions,

$$
\begin{aligned}
\mid\left(p_{3 / 2}\right)^{3} ; J^{\pi} & \left.=3 / 2_{1}^{-}\right\rangle, & E_{0} & =(2.26-0.98 i) \mathrm{MeV}, \\
\mid p_{1 / 2}\left(p_{3 / 2}\right)_{0}^{2} ; J^{\pi} & \left.=1 / 2_{1}^{-}\right\rangle, & E_{0} & =(3.66-3.57 i) \mathrm{MeV}, \\
\mid p_{1 / 2}\left(p_{3 / 2}\right)_{2}^{2} ; J^{\pi} & \left.=3 / 2_{2}^{-}\right\rangle, & & E_{0}=(3.66-3.57 i) \mathrm{MeV}, \\
\mid p_{1 / 2}\left(p_{3 / 2}\right)_{2}^{2} ; J^{\pi} & \left.=5 / 2_{1}^{-}\right\rangle, & E_{0} & =(3.66-3.57 i) \mathrm{MeV}, \\
\mid\left(p_{1 / 2}\right)_{0}^{2} p_{3 / 2} ; J^{\pi} & \left.=3 / 2_{3}^{-}\right\rangle, & E_{0} & =(5.06-6.15 i) \mathrm{MeV} .
\end{aligned}
$$

We report here only the converged results, which give the ${ }^{7} \mathrm{He}$ resonances,

$$
\begin{aligned}
& E\left(3 / 2_{1}^{-}\right)=(0.02-0.08 i) \mathrm{MeV}, \\
& E\left(1 / 2_{1}^{-}\right)=(0.39-3.98 i) \mathrm{MeV}, \\
& E\left(3 / 2_{2}^{-}\right)=(2.43-1.95 i) \mathrm{MeV}, \\
& E\left(5 / 2_{1}^{-}\right)=(2.75-0.89 i) \mathrm{MeV}, \\
& E\left(3 / 2_{3}^{-}\right)=(3.85-3.06 i) \mathrm{MeV} .
\end{aligned}
$$

For the $J^{\pi}=3 / 2^{-}$case, which has the most severe dimensionality, the converged values reported above were obtained with a reference space of dimension $N_{P^{\prime}} \approx 1400-1800$. So for the $J^{\pi}=3 / 2^{-}$case, the dimension has been drastically reduced from 38896 to $1400-1800$. Figure 17 shows a plot of the calculated energy levels for the nuclei ${ }^{5-7} \mathrm{He}$ within our truncation procedure. The noninteracting energy levels for ${ }^{6} \mathrm{He}$ and ${ }^{7} \mathrm{He}$ are also shown and serve to illustrate how the two- and three-particle resonances develop when the nucleon-nucleon interaction from Eq. (16) is turned on.

There are several interesting features to be seen from Fig. 17. The $0^{+}$and $2^{+}$states in ${ }^{6} \mathrm{He}$ are formed within our model because of a strong pairing effect between the two neutrons moving in equivalent orbits. Thus, when the nucleon-nucleon interaction is turned on, we observe that the $0_{1}^{+}$and $2_{1}^{+}$states drop drastically in energy, and one becomes bound and the other almost bound. On the other hand, the $\left(p_{1 / 2} \otimes p_{3 / 2}\right) 1^{+}$state in ${ }^{6} \mathrm{He}$ exhibits weak pairing effects. Our preliminary calculation of ${ }^{7} \mathrm{He}$ exhibits a richer continuum structure than so far seen in experiment (see the review [22] and references therein). In Ref. [22], two excited states with tentative spins $J^{\pi}=1 / 2^{-}$and $J^{\pi}=5 / 2^{-}$are reported to exist above the ${ }^{7} \mathrm{He}$ ground state, at excitation energies 0.57 and $2.87 \mathrm{MeV}$, respectively. The main decay channel of the resonance at $2.87 \mathrm{MeV}$ is $\alpha+3 n$. From this decay channel, Ref. [22] concludes that the configuration $\left|p_{1 / 2}\left(p_{3 / 2}\right)_{2}^{2} ; J^{\pi}=5 / 2^{-}\right\rangle$, involving the ${ }^{6} \mathrm{He} 2_{1}^{+}$state, is the most probable one, which is also our finding. The $J^{\pi}=1 / 2^{-}$

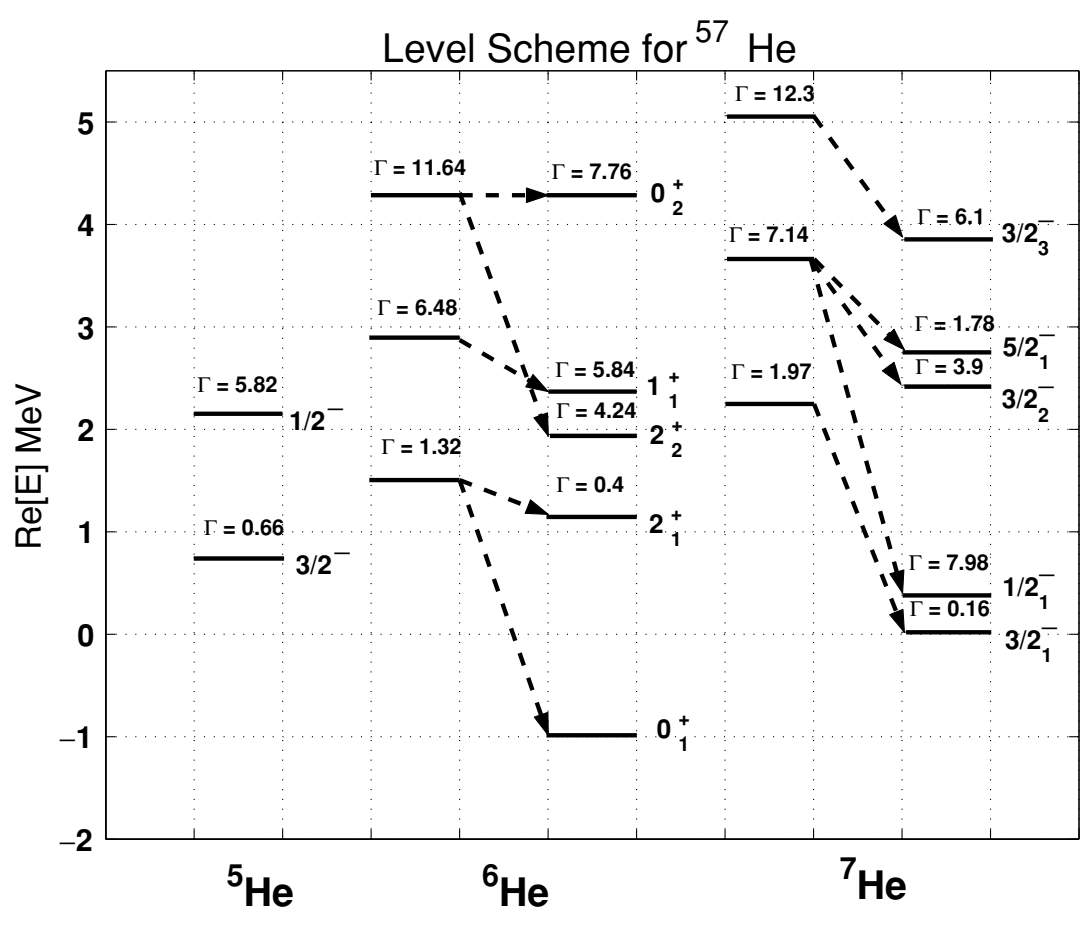

FIG. 17. Energy levels for ${ }^{6} \mathrm{He}$ and ${ }^{7} \mathrm{He}$ for interacting and noninteracting valence neutrons, based on our computed ${ }^{5} \mathrm{He}$ single-particle spectrum, including both $p_{1 / 2}$ and $p_{3 / 2}$ orbits. The noninteracting energy levels for ${ }^{6} \mathrm{He}$ and ${ }^{7} \mathrm{He}$ are shown to the left in each case. 
resonance exhibits a peculiar behavior; it moves way down toward the threshold in real energy when the nucleon-nucleon interaction is turned on; on the other hand, the width increases slightly. However, these results must be gauged with the fact that we are using a purely phenomenological nucleon-nucleon interaction model. The inclusion of a realistic interaction is the topic for future work. The $1 / 2^{-}$state is certainly not a simple spin-orbit partner to the $3 / 2^{-}$ground state. We will return to a comprehensive discussion of the ${ }^{7} \mathrm{He}$ spectrum in a forthcoming paper, making comparisons with other procedures (see, for example, Ref. [43]). A realistic calculation has also to account for the recoil of the $\alpha$ particle. However, the main issue here was to demonstrate how to derive effective interactions for the Gamow shell model, with a considerable reduction in dimensionality.

\section{CONCLUSION AND FUTURE PERSPECTIVES}

In this work, we applied the contour deformation method in momentum space, with a single-particle basis in momentum space serving as the starting point for Gamow shell-model calculations of loosely bound nuclei. Our main purpose was to propose an effective interaction scheme for Gamow shellmodel calculations. One of the most severe difficulties regarding these calculations is the dramatic growth of dimension when dealing with several valence particles moving in a large shell-model space. This dimensionality problem is even more severe than in the harmonic oscillator representation used in traditional shell-model equation studies. In the Berggren representation, a large number of complex-continuum orbits has to be included as well. The clear distinction of the noninteracting resonances from the dense distribution of complexcontinuum states allows use of a perturbation treatment, when configuration mixing is taken into account. For perturbation expansions to converge, the unperturbed states have to be well separated from the $Q$-space states, or else the propagators will contain poles that make a perturbative treatment difficult. We have shown that the Lee-Suzuki similarity transformation combined with the multireference perturbation method reduces the necessary basis to about $3-4 \%$ that of the full problem.

To test the procedure, the resonant spectra of the dripline nuclei ${ }^{5-7} \mathrm{He}$ have been studied and described using phenomenologically derived nucleon-nucleon interactions. We have shown that our choice of contour gives a good convergence for various resonant multinucleon states, and in addition it allows a clear distinction to be made between physical states and the remaining complex-continuum states.

Treating the many-particle problem in a perturbation scheme requires finding a reference (model) space containing most of the many-body correlations. The method and scheme outlined here allow a perturbative treatment of many-body states in which antibound states also play an important role, such as in the drip-line nucleus ${ }^{11} \mathrm{Li}$.

The location and width of multiparticle resonances also depend on the effective interaction used between valence nucleons. The next step is to derive a realistic effective interaction for Gamow shell-model calculations, and self- consistent Hartree-Fock single-particle energies for loosely bound nuclei, starting from a realistic nucleon-nucleon force. Using the Berggren representation may give an underlying understanding of many-body resonances from a microscopic point of view. Moreover, in our algorithm of Sec. V we employed the multireference perturbation method. Our future plans involve replacing the perturbative treatment by the more flexible coupled cluster approaches, as discussed in Refs. [40,41]. The substantial challenge of computing other observables than energy is also being addressed, with inspirations drawn from few-body studies using hyperharmonics $[44,45]$.

\section{ACKNOWLEDGMENTS}

Support by the Research Council of Norway is gratefully acknowledged. G.H. also gratefully acknowledges support by the Centre of Mathematics for Applications at the University of Oslo. Discussions with M. Kartamyshev, B. V. Danilin, and S. N. Ershov were very helpful.

\section{APPENDIX: THREE-BODY MATRIX ELEMENTS IN $\boldsymbol{j}-\boldsymbol{j}$ COUPLING}

The matrix element of a two-body interaction with antisymmetric three-body Berggren states in $j-j$ coupling may be written in terms of antisymmetric two-body matrix elements as

$$
\begin{aligned}
& \left\langle\tilde{\Phi}_{(a b) c}(123)|V| \Psi_{(d e) f}(123)\right\rangle \\
& =\langle\tilde{a b}|v| d e\rangle_{J_{a b}}^{A S} \delta_{c, f} \delta_{J_{a b}, J_{d e}}+\left(\frac{1+\delta_{d, f}}{1+\delta_{d, e}}\right)^{1 / 2} \\
& \times(-1)^{j_{d}-J_{d e}+J_{a b}-J} U\left(j_{e} j_{d} J_{j} ; J_{d e} J_{a b}\right)\langle\tilde{a b}|v| d f\rangle_{J_{a b}}^{A S} \delta_{c, e} \\
& -\left(\frac{1+\delta_{e, f}}{1+\delta_{d, e}}\right)^{1 / 2}(-1)^{j_{d}+J_{a b}-J} U\left(j_{d} j_{e} J j_{f} ; J_{d e} J_{a b}\right) \\
& \times\langle\tilde{a b}|v| e f\rangle_{J_{a b}}^{A S} \delta_{c, d}+\left(\frac{1+\delta_{b, c}}{1+\delta_{a, b}}\right)^{1 / 2}(-1)^{j_{a}+J_{d e}-J} \\
& \times U\left(j_{a} j_{b} J j_{c} ; J_{a b} J_{d e}\right)\langle\tilde{b c}|v| d e\rangle_{J_{d e}}^{A S} \delta_{a, f}+\left(\frac{1+\delta_{b, c}}{1+\delta_{a, b}}\right)^{1 / 2} \\
& \times \sum_{J_{b c}}(-1)^{j_{a}+j_{d}-2 J} U\left(j_{a} j_{b} J j_{c} ; J_{a b} J_{b c}\right) \times\left\{\left(\frac{1+\delta_{d, f}}{1+\delta_{d, e}}\right)^{1 / 2}\right. \\
& \times(-1)^{J_{d e}} U\left(j_{e} j_{d} J_{j} ; J_{d e} J_{b c}\right)\langle\tilde{b c}|v| d f\rangle_{J_{b c}}^{A S} \delta_{a, e} \\
& \left.+\left(\frac{1+\delta_{e, f}}{1+\delta_{d, e}}\right)^{1 / 2} U\left(j_{d} j_{e} J_{j} ; J_{d e} J_{b c}\right)\langle\tilde{b c}|v| e f\rangle_{J_{b c}}^{A S} \delta_{a, d}\right\} \\
& +\left(\frac{1+\delta_{a, c}}{1+\delta_{a, b}}\right)^{1 / 2}(-1)^{j_{a}-J_{a b}+J_{d e}-J} U\left(j_{b} j_{a} J j_{c} ; J_{a b} J_{d e}\right) \\
& \times\langle\tilde{a c}|v| d e\rangle_{J_{d e}}^{A S} \delta_{b, f}+\left(\frac{1+\delta_{a, c}}{1+\delta_{a, b}}\right)^{1 / 2} \sum_{J_{a c}}(-1)^{j_{a}+j_{d}-J_{a b}-2 J}
\end{aligned}
$$




$$
\begin{aligned}
& \times U\left(j_{b} j_{a} J_{j} ; J_{a b} J_{a c}\right)\left\{\left(\frac{1+\delta_{d, f}}{1+\delta_{d, e}}\right)^{1 / 2}(-1)^{J_{d e}}\right. \\
& \times U\left(j_{e} j_{d} J_{j} ; J_{d e} J_{a c}\right)\langle\tilde{a c}|v| d f\rangle_{J_{a c}}^{A S} \delta_{b, e}+\left(\frac{1+\delta_{e, f}}{1+\delta_{d, e}}\right)^{1 / 2} \\
& \left.\times U\left(j_{d} j_{e} J_{f} ; J_{d e} J_{a c}\right)\langle\tilde{a c}|v| e f\rangle_{J_{a c}}^{A S} \delta_{b, d}\right\}
\end{aligned}
$$

Here $U\left(j_{a}, j_{b}, J, j_{c} ; J_{a b}, J_{b c}\right)$ is the normalized Racah coefficient, and $\langle\tilde{a} b|=\left\langle a b^{*}\right|$ is due to the Berggren metric.

In the case where all the single-particle orbits in the ket (bra) are equivalent, i.e., $d=e=f$, one needs coefficients of fractional parentage in order to make the three-body wave function totally antisymmetric in $j-j$ coupling. The antisymmetric three-body matrix element then takes the form,

$$
\begin{aligned}
\left\langle\tilde{\Phi}_{(a b) c}\right. & \left.(123)|V| \Phi_{d d d}(123)\right\rangle \\
= & \left.\left.\sqrt{3}\left\langle j_{d}^{2} J_{a b}, j_{d}\right|\right\} j_{d}^{3} J\right\rangle\langle\tilde{a b}|v| d d\rangle_{J_{a b}}^{A S} \delta_{c, d} \\
+ & \left.\left.\sqrt{3}(-1)^{j_{a}+j_{d}-2 J} \sum_{K}\left\langle j_{d}^{2} K, j_{d}\right|\right\} j_{d}^{3} J\right\rangle \\
\times & \left\{\left(\frac{1+\delta_{b, c}}{1+\delta_{a, b}}\right)^{1 / 2} \sum_{J_{b c}} U\left(j_{a} j_{b} J j_{c} ; J_{a b} J_{b c}\right)\right. \\
& \times U\left(j_{d} j_{d} J j_{d} ; K J_{b c}\right)\langle\tilde{b c}|v| d d\rangle_{J_{b c}} \delta_{a, d} \\
& +\left(\frac{1+\delta_{a, c}}{1+\delta_{a, b}}\right)^{1 / 2} \sum_{J_{a c}}(-1)^{J_{a b}} U\left(j_{b} j_{a} J j_{c} ; J_{a b} J_{a c}\right) \\
& \left.\times U\left(j_{d} j_{d} J j_{d} ; K J_{a c}\right)\langle\tilde{a c}|v| d d\rangle_{J_{b c}} \delta_{b, d}\right\}
\end{aligned}
$$

where $\left.\left.\left\langle j_{d}^{2} J_{a b}, j_{d}\right|\right\} j_{d}^{3} J\right\rangle$ is the coefficient of fractional parentage.
[1] N. Michel, W. Nazarewicz, and M. Płoszajczak, Phys. Rev. C 70, 064313 (2004).

[2] N. Michel, W. Nazarewicz, M. Płoszajczak, and J. Rotureau, nucl-th/0401036 (2004).

[3] J. Dobaczewski, W. N. N. Michel, M. Płoszajczak, and M. V. Stoitsov, nucl-th/0401034 (2004).

[4] R. J. Liotta, E. Maglione, N. Sandulescu, and T. Vertse, Phys. Lett. B367, 1 (1996).

[5] R. IdBetan, R. J. Liotta, N. Sandulescu, and T. Vertse, Phys. Rev. C 67, 014322 (2003).

[6] N. Michel, W. Nazarewicz, M. Płoszajczak, and K. Bennaceur, Phys. Rev. Lett. 89, 042502 (2002).

[7] N. Michel, W. Nazarewicz, M. Płoszajczak, and J. Okołowicz, Phys. Rev. C 67, 054311 (2003).

[8] R. IdBetan, R. J. Liotta, N. Sandulescu, and T. Vertse, Phys. Rev. Lett. 89, 042501 (2002).

[9] R. IdBetan, R. J. Liotta, N. Sandulescu, and T. Vertse, Phys. Lett. B584, 48 (2004).

[10] K. Bennaceur, F. Nowacki, J. Okołowicz, and M. Płoszajczak, Nucl. Phys. A651, 289 (1999).

[11] K. Bennaceur, F. Nowacki, J. Okołowicz, and M. Płoszajczak, Nucl. Phys. A671, 203 (2000).

[12] J. Okołowicz, M. Płoszajczak, and I. Rotter, Phys. Rep. 374, 271 (2003).

[13] A. Volya and V. Zelevinsky, Phys. Rev. C 67, 054322 (2003).

[14] T. Berggren, Nucl. Phys. A109, 265 (1968).

[15] T. Berggren, Nucl. Phys. A169, 353 (1971).

[16] T. Berggren, Phys. Lett. B73, 389 (1978).

[17] T. Berggren, Phys. Lett. B373, 1 (1996).

[18] P. Lind, Phys. Rev. C 47, 1903 (1993).

[19] G. Hagen, J. S. Vaagen, and M. Hjorth-Jensen, J. Phys. A: Math. Gen. 37, 8991 (2004).

[20] E. R. Davidson, Comput. Phys. Commun. 53, 49 (1989).

[21] E. R. Davidson, Comput. Phys. 5, 519 (1993).

[22] B. Jonson, Phys. Rep. 389, 1 (2004).

[23] M. V. Zhukov, B. V. Danilin, D. V. Fedorov, J. M. Bang, I. J. Thompson, and J. S. Vaagen, Phys. Rep. 231, 151 (1993).
[24] N. Moiseyev, Phys. Rep. 302, 211 (1998).

[25] R. R. Whitehead, A. Watt, B. J. Cole, and I. Morrison, Adv. Nucl. Phys., edited by M. Baranger and E. Vogt (Plenum, New York, 1977), Vol. 9, p. 123.

[26] K. Suzuki and S. Y. Lee, Prog. Theor. Phys. 64, 2091 (1980).

[27] K. Suzuki, Prog. Theor. Phys. 68, 246 (1982).

[28] K. Suzuki and R. Okamoto, Prog. Theor. Phys. 93, 905 (1995).

[29] S. Fujii, E. Epelbaum, H. Kamada, R. Okamoto, K. Suzuki, and W. Glöckle, Phys. Rev. C 70, 024003 (2004).

[30] C. Buth, R. Santra, and L. S. Cederbaum, Phys. Rev. A 69, 032505 (2004).

[31] F. Chen, E. R. Davidson, and S. Iwata, Int. J. Quantum Chem. 86, 256 (2002).

[32] R. Santra and L. S. Cederbaum, Phys. Rep. 368, 1 (2002).

[33] S. Sack, L. C. Biedenharn, and G. Breit, Phys. Rev. 93, 321 (1954).

[34] P. Navrátil and B. R. Barrett, Phys. Rev. C 57, 562 (1998).

[35] P. Navrátil, J. P. Vary, and B. R. Barrett, Phys. Rev. Lett. 84, 5728 (2000).

[36] P. Navrátil, J. P. Vary, and B. R. Barrett, Phys. Rev. C 62, 054311 (2000).

[37] P. Navrátil, G. P. Kamuntavicius, and B. R. Barrett, Phys. Rev. C 61, 044001 (2000).

[38] N. J. Higham, Numer. Algorithms 15, 227 (1997).

[39] E. D. Denman and A. N. Beavers, Appl. Math. Comput. 2, 63 (1976).

[40] D. J. Dean and M. Hjorth-Jensen, Phys. Rev. C 69, 054320 (2004).

[41] K. Kowalski, D. J. Dean, M. Hjorth-Jensen, T. Papenbrock, and P. Piecuch, Phys. Rev. Lett. 92, 132501 (2004).

[42] E. R. Davidson, E. Engdahl, and N. Moiseyev, Phys. Rev. A 33, 2436 (1986).

[43] S. C. Pieper, R. B. Wiringa, and J. Carlson, Phys. Rev. C 70, 054325 (2004); nucl-th/0409012 (2004).

[44] S. N. Ershov, B. V. Danilin, and J. S. Vaagen, Phys. Rev. C 64, 064609 (2001).

[45] B. V. Danilin, T. Rogde, J. S. Vaagen, I. J. Thompson, and M. V. Zhukov, Phys. Rev. C 69, 024609 (2004). 